\title{
ARTICLE
}

\section{CID1067700, a late endosome GTPase Rab7 receptor antagonist, attenuates brain atrophy, improves neurologic deficits and inhibits reactive astrogliosis in rat ischemic stroke}

\author{
Yuan Qin ${ }^{1}$, Yang $\mathrm{He}^{1}$, Yong-ming $\mathrm{Zhu}^{1}$, Min $\mathrm{Li}^{1}$, Yong $\mathrm{Ni}^{1}$, Jin $\mathrm{Liu}^{1}$ and Hui-ling Zhang ${ }^{1}$
}

\begin{abstract}
Increasing evidence suggests that Ras-related in brain 7 (Rab7), an endosome-localized small GTPase contributes to cerebral ischemic brain injury. In the present study, we investigated the role of Rab7 in ischemic stroke-induced formation of astrogliosis and glial scar. Rats were subjected to transient middle cerebral artery occlusion (tMCAO); the rats were injected with the Rab7 receptor antagonist CID1067700 (CID). Primary astrocytes were subjected to an oxygen and glucose deprivation and reoxygenation $(\mathrm{OGD} / \mathrm{Re})$ procedure; CID was added to the cell culture media. We found that Rab7 was significantly elevated over time in both the in vivo and in vitro astrocytic injury models, and administration of CID significantly down-regulated the glial scar markers such as glial fibillary acidic protein (GFAP), neurocan and phosphacan. Moreover, administration of CID significantly attenuated the brain atrophy and improved neurologic deficits in tMCAO rats, and protected astrocytes against OGD/Re-induced injury. Further, CID downregulated the protein levels of Lamp1 and active cathepsin B in astrocytes after OGD/Re or tMCAO injury; CID inhibited the co-localization of cathepsin B and Rab7, Lamp1 and Rab7; CID decreased OGD/Re-induced increase in lysosomal membrane permeability and blocked OGD/Re-induced release of cathepsin B from the lysosome into the cytoplasm in astrocytes. Taken together, these results suggest that Rab7 is involved in ischemic stroke-induced formation of astrogliosis and glial scar. CID administration attenuates brain atrophy and improves neurologic deficits and inhibits astrogliosis and glial scar formation after ischemic stroke via reducing the activation and release of cathepsin B from the lysosome into the cytoplasm.
\end{abstract}

Keywords: ischemic stroke; astrocytes; Rab7; CID1067700; glial scar; lysosome

Acta Pharmacologica Sinica (2019) 40:724-736; https://doi.org/10.1038/s41401-018-0166-8

\section{INTRODUCTION}

Ischemic stroke is a leading cause of combined death and disability worldwide [1]. At present, tissue plasminogen activator (t-PA) is the only thrombolytic agent approved by the United States Food and Drug Administration for the treatment of acute ischemic stroke [2]. However, the use of tPA is limited to a minority of patients due to its narrow therapeutic window and side effects $[3,4]$. Therefore, the development of novel targets for drug therapies to reduce brain damage after ischemic stroke is a major challenge [5].

The central nervous system contains an abundance of astrocytes [6]. Astrocytes play important roles in many diseases and respond to most forms of neural disorders, including ischemic stroke [7]. Glial fibrillary acidic protein (GFAP) is a prototypic marker for reactive astrocytes [8]. In the acute phase of ischemic stroke, reactive astrocytes in the peri-infarct region seal the site of injury, protect the damaged neural tissue, prevent an overwhelming inflammatory response, and re-establish the blood-brain barrier [9-12]. However, a physical and functional wall is formed around the necrotic brain tissue of the infarct during the chronic phase, which occurs approximately 2-4 weeks after ischemic stroke [10, 13]. Reactive astrocytes in the scar express a broad range of inhibitory molecules against axonal regeneration, such as chondroitin sulfate proteoglycans (CSPGs) $[14,15]$, which are major barriers to axon extension and regeneration in the central nervous system [16]. Therefore, the inhibition of glial scar formation may be a therapeutic strategy for ischemic stroke [17].

Previous studies demonstrated that lysosomal alterations such as lysosomal membrane permeabilization (LMP) and the release of lysosomal hydrolytic enzymes occur in cerebral ischemia diseases [6, 18-20]. Lysosomal membranes contain two highly glycosylated proteins, Lamp1 and Lamp2, as major components [21]. Cathepsin B is one of the major lysosomal cysteine protease that might be important in intracellular protein catabolism and apoptosis induction [6]. The release of active cathepsin B from the lysosomes to the cytoplasm can be used as a measure of lysosomal membrane permeability in ischemic neurons and astrocytes [6, 22]. Our previous study reported a significant upregulation of Lamp1 protein in astrocytes after tMCAO, which coincided with increased levels of active cathepsin B and its movement into the cytosol. Furthermore, administration of a

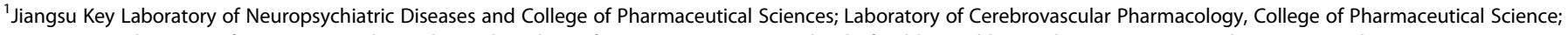
Jiangsu Key Laboratory of Preventive and Translational Medicine for Geriatric Diseases, School of Public Health, Soochow University, Suzhou 215123, China Correspondence: Hui-ling Zhang (zhanghuiling@suda.edu.cn)

These authors contributed equally: Yuan Qin, Yang He
}

Received: 21 April 2018 Accepted: 29 August 2018

Published online: 12 October 2018 
cathepsin B inhibitor [6, 22] blocked LMP in ischemic astrocytes and attenuated astrocytic cell death, which suggests that a similar approach might hold therapeutic potential for the treatment of diseases associated with LMP, including ischemic stroke [23].

Rab7 is a 208 amino acid protein predominantly found in the late endosomes [24]. Rab7 is recognized as the only lysosomal Rab protein in the Rab GTPase superfamily [25]. Late endosomes are a central hub that receive the following factors: (i) newly synthesized proteins (e.g., cathepsins) from the Golgi apparatus, (ii) endocytic cargo from the early endosome, and (iii) autophagic cargo from the autophagosome. Late endosomes fuse with lysosomes via an N-ethylmaleimide-sensitive factor-dependent mechanism to become a more acidic hybrid endolysosome, also known as the secondary lysosome, for component degradation. The endolysosome becomes a lysosome with evenly distributed contents after digestion of the cargo. The lysosome is recycled for another round of fusion with late endosomes for degradation. Previous research [26] revealed that a massive buildup of damaged late endosomes occurs over time in nerve cells, which die after transient cerebral or global ischemia. However, the role of Rab7 in astrogliosis and glial scar formation is largely unknown. In this study, we aimed to investigate the role of Rab7 in reactive astrocytes during the course of glial scar formation and its underlying mechanisms associated with a reduction in cathepsin B activation and release from the lysosomes into the cytoplasm after ischemic stroke. We report that (i) Rab7 is significantly elevated in the reactive astrocytes in the peri-infarct area of the cerebral cortex following tMCAO and that a positive correlation exists between Rab7 upregulation and astrogliosis and glial scar formation; (ii) the Rab7 receptor antagonist CID1067700 (CID) reduces astrogliosis and glial scar formation; (iii) CID promotes brain functional recovery in the late stage of ischemic stroke; and (iv) CID attenuates astrogliosis and glial scar formation after ischemic stroke via a reduction of cathepsin B activation and release from the lysosomes into the cytoplasm.

\section{MATERIALS AND METHODS}

Animals and treatment

Male Sprague-Dawley (SD) rats weighing (280-320 g) were purchased from the SLAC Company (Shanghai, China). The University Committee on Animal Care of Soochow University approved all animal procedures. Rats were housed in groups of four per cage in a standard animal room with a $12 \mathrm{~h}$ light $/ 12 \mathrm{~h}$ dark cycle at $22^{\circ} \mathrm{C}$ and given free access to food and water. All procedures were designed to minimize suffering and the number of animals used.

Middle cerebral artery occlusion and reperfusion

Transient focal cerebral ischemia was induced via intraluminal occlusion of the right middle cerebral artery (MCA), as previously described [6]. Rats were randomly assigned to groups using the online tool Quickcalcs (http://www.graphpad.com/quickcalcs/). Rats received an intraperitoneal injection of $4 \%$ choral hydrate $(350 \mathrm{mg} / \mathrm{kg})$, and the right common carotid artery (CCA) was exposed via a midline pretracheal incision. The external carotid artery (ECA) and CCA were ligated. A rubber silicon-coated monofilament suture (nylon monofilament size 4-0, diameter $0.150-0.199 \mathrm{~mm}$, length $28 \mathrm{~mm}$; diameter with coating $0.36 \pm 0.02$ $\mathrm{mm}$; coating length $5 \mathrm{~mm}$ ) was inserted into the ECA and advanced along the internal carotid artery $8-10 \mathrm{~mm}$ from the bifurcation of the carotid artery to occlude the MCA. The suture was gently withdrawn $90 \mathrm{~min}$ after ischemia for reperfusion. Tympanic membrane and rectal temperature probes were inserted, and cranial and body temperatures were maintained at $37^{\circ} \mathrm{C}$ using a heating pad during and after surgery until recovery from anesthesia. CID 1067700 (CID) was purchased from Merck Millipore (Temecula, California, USA). CID ( $2.5 \mathrm{mg} / \mathrm{kg}$ dissolved in
$2 \%$ DMSO) or vehicle (2\% DMSO) was administered intraperitoneally 10 min before tMCAO, 90 min after the onset of tMCAO, and once weekly for four weeks. Sham-operated and tMCAO control animals received intraperitoneal injections of vehicle.

Assessment of neurological deficits and brain atrophy Neurological deficits score, grip strength test and limb-useasymmetry test were used to assess neurological deficits, as described previously [22]. The tests were performed before and 1, $3,7,14,21,28$ days after tMCAO. Brain atrophy was measured 28 days after tMCAO. Observers who were blind to group assignment performed all evaluations.

Neurological deficits score. Animals were scored on a 6-point scale for neurological deficits: 0 , no neurological deficit performance; 1 , ptosis of eyelid ipsilateral to the occluded MCA side and/or failure to stretch ipsilateral forelimb; 2, persistently walks toward the ipsilateral side in large circles; 3, persistently walks toward ipsilateral side in small circles and/or rolls over repeatedly; 4 , lies nearly motionless on the contralateral side; and 5, died after recovery from anesthesia.

Grip strength test. A rat grip strength meter (YLS-13A, Jinan YiYan Technology Development Company Limited, China) was used to evaluate forelimb grip strength. The peak force was recorded as the grip value (grams as the unit of force), and 10 trials were performed for each animal.

Limb-use-asymmetry test. A Plexiglas cylinder (HOOFAN, Wenling, Zhejiang) was used to test forelimb-use-asymmetry. Forelimb movement was recorded while the rats moved vertically along the wall of the cylinder. A total of twenty movements were recorded during the $10 \mathrm{~min}$ test for each rat. The final score was calculated as follows: (Right forelimb movement - Left forelimb movement) / (Right forelimb movement + Left forelimb movement + Both movements).

Brain atrophy measurement. Brain atrophy measurement was performed as described previously [27]. Twenty-eight days after tMCAO, behavioral testing was performed, and then the rats were euthanized. Brains were dissected and sliced into five parts in a plastic module (Harvard Apparatus, 3-mm thickness) to observe the volume of brain atrophy. The ratio of brain atrophy (RA) was analyzed using Image J software. The atrophy volume was measured as follows: RA (ratio of brain atrophy, $\%$ of the left brain) $=(\mathrm{LT}-\mathrm{RT}) / \mathrm{LT} \times 100 \%)$, where $\mathrm{LT}=$ total volume in the left hemisphere, and $\mathrm{RT}=$ total volume in the right hemisphere.

Primary cell cultures, oxygen-glucose deprivation/reperfusion (OGD/Re) and lactate dehydrogenase (LDH) leakage measurement Primary cortical astrocyte cultures. Primary cortical astrocyte cultures were prepared from $24 \mathrm{~h}$ postnatal Sprague-Dawley rats as described previously [22]. Briefly, cerebral cortices were digested with $2.5 \%$ trypsin for $10 \mathrm{~min}$ at $37^{\circ} \mathrm{C}$ and filtered through a sterile $40-\mu \mathrm{m}$ nylon cell strainer. Cells were cultured in DMEM/F12 (GIBCO, 11330) containing 10\% fetal bovine serum (GIBCO, 10099) and $1 \% 100 \mathrm{U} / \mathrm{mL}$ penicillin/streptomycin (Beyotime, C0222). Cultures were maintained at $37^{\circ} \mathrm{C}$ under $>90 \%$ humidity and $5 \% \mathrm{CO}_{2}$. The medium was changed every 2 or 3 days until cells were confluent. More than $90 \%$ of the cultured cells were astrocytes, as identified by immunofluorescent staining for the astrocytic marker protein GFAP.

Oxygen-glucose deprivation/reperfusion (OGD/Re). The model of oxygen and glucose deprivation (OGD) was performed as described previously [28]. Briefly, primary cultured astrocytes were rinsed twice with phosphate-buffered saline (PBS, $\mathrm{pH} 7.4$ ) and refreshed with glucose-free DMEM (GIBCO, 11966). Cells were 
placed in a sealed chamber (Billups-Rothenberg, San Diego, CA, USA) loaded with mixed gas containing $95 \% \mathrm{~N}_{2}$ and $5 \% \mathrm{CO}_{2}$ at $37^{\circ} \mathrm{C}$ for $6 \mathrm{~h}$. After OGD exposure, the cells were subjected to reoxygenation in a DMEM/F12 solution in normoxic conditions for $12 \mathrm{~h}$ and $24 \mathrm{~h}$. Cells in the control group (non-OGD) were incubated in a DMEM/F12 solution in a humidified atmosphere with $5 \% \mathrm{CO}_{2}$ at $37^{\circ} \mathrm{C}$ for the same time periods as the experimental groups. CID was added to the cell culture media $30 \mathrm{~min}$ before OGD and reperfusion. CID was dissolved in DMSO, and the final concentrations in the cell culture media were 40 and $80 \mathrm{nM}$. The DMSO concentration during the cell culture experiments never exceeded $0.1 \%$.

Lactate dehydrogenase (LDH) leakage measurement. LDH leakage from astrocytes was detected using an LDH assay kit (Nanjing Jiancheng Bioengineering Institute, Nanjing, China) at $450 \mathrm{~nm}$ in an automatic multiwall spectrophotometer (Bio-Rad Laboratories, Hercules, CA, USA). The method was performed as previously described [28]. Briefly, the medium was collected after reperfusion. Astrocytes were rinsed with PBS and lysed in 1\% TritonX-100 at 37 ${ }^{\circ} \mathrm{C}$ for $30 \mathrm{~min}$. Samples of the media and cell lysates were prepared following the manufacturer's instructions for the LDH assay. The LDH leakage was calculated as follows: LDH leakage (\%) $=\mathrm{OD}$ value of the supernatant of the medium/(OD value of the supernatant of the medium $+O D$ value of the supernatant of lysed cells) $\times 100 \%[29]$.

Immunohistochemistry and immunofluorescence

Immunohistochemistry was performed as previously described [6]. Rats were euthanized using $4 \%$ chloral hydrate $(350 \mathrm{mg} / \mathrm{kg}) 1,3$, and 7 days after tMCAO. Brains were perfused and fixed in $4 \%$ paraformaldehyde for $24 \mathrm{~h}$, dehydrated in increasing concentrations of saccharose, embedded in OCT (optimal cutting temperature compound, Sakura, USA), and cut into $12 \mu \mathrm{m}$ thick sections in the coronal plane. Brain sections were fixed in 4\% paraformaldehyde for $10 \mathrm{~min}$, permeabilized with $0.3 \%$ Triton X-100 and blocked in $1 \%$ BSA for $1 \mathrm{~h}$ at room temperature. Slices were incubated with specific primary antibodies against GFAP (1:1000, ab10062, Abcam), GFAP (1:500, ab7260, Abcam), Rab7 (1:500, ab77993, Abcam), and Lamp1 (1:500, ab25630, Abcam) overnight at $4{ }^{\circ} \mathrm{C}$. The corresponding secondary antibodies of Alexa Fluor ${ }^{\circledR}$ 488 goat anti-rabbit $\lg G(H+L)(1: 500, A 11008$, Life Technologies) and Alexa Fluor ${ }^{\circledR} 594$ goat anti-mouse $\lg G(H+L)$ (1:500, A11005, Life Technologies) were applied for $1 \mathrm{~h}$ at room temperature. Sections were incubated for $20 \mathrm{~min}$ in Hoechst $(1: 10,000,33258$, Sigma) solution to stain nuclei.

Cultured cells were treated with OGD for $6 \mathrm{~h}$ and reoxygenation for $24 \mathrm{~h}$. Cells were rinsed with PBS and fixed in 4\% paraformaldehyde for cell morphology assessments. Cells were then permeabilized and blocked for $60 \mathrm{~min}(0.1 \%$ Triton X-100, $1 \%$ BSA) at room temperature and overnight at $4{ }^{\circ} \mathrm{C}$ with the following primary antibodies: GFAP (1:500, AB5804, Millipore), GFAP (1:500, MAB360, Millipore), neurocan (1:200, ab26003, Abcam), phosphacan (1:500, P8874, Sigma), Lamp1 (1:100, ab24170, Abcam), Rab7 (1:500, ab50533, Abcam) and cathepsin B (1:100, 06-480, Millipore). Cells were subsequently incubated for $1 \mathrm{~h}$ with the corresponding secondary antibodies at room temperature. Cells were washed in PBS and incubated in Hoechst $(1: 10,000$, 33258, Sigma) solution for $10 \mathrm{~min}$. Images were obtained by confocal laser scanning microscopy (LSM 710; Carl Zeiss Co. Ltd., Oberkochen, Germany). We used ZEN 2012 and ImageJ to analyze immunofluoresence.

Two colocalization coefficients (Pearson's overlap coefficients and Mander's overlap coefficients) were used to express the intensity correlation of colocalized objects in each component of a dual-color image. Pearson's overlap coefficients indicate the correlation of the intensity distribution in each color channel. The results are +1 for perfect correlation, 0 for no significant correlation, and -1 for complete anti-correlation. This coefficient only measures correlations in colocalization experiments. Mander's overlap coefficients indicate an actual overlap between color channels, which represents the true level of colocalization. Values range from 0 to 1 , and the results are 1 for perfect overlap, and 0 for no overlap. It is similar to Pearson's overlap coefficients in the colocalization experiments, but the difference is that Mander's overlap coefficients are suitable when the fluorescence of one antigen is stronger than another antigen [30].

\section{Western blotting analysis}

Proteins extracted from ischemic cortexes or cultured astrocytes were used for Western blotting. Tissues and cells were lysed in lysis buffer $(10 \mathrm{mM}$ Tris- $\mathrm{HCl}, 150 \mathrm{mM} \mathrm{NaCl}, 1 \%$ TritonX-100, 1\% sodium deoxycholate, $0.1 \%$ sodium dodecyl sulfate, $5 \mathrm{mM}$ EDTA, pH 7.4) containing a protease inhibitor cocktail, incubated for $30 \mathrm{~min}$ on ice, and centrifuged at $12,000 \times g$ for $10 \mathrm{~min}$ at $4{ }^{\circ} \mathrm{C}$. The protein concentration of the supernatant was determined by BCA Protein Assay Kit (Pierce, Rockford, IL, USA). Protein $(40 \mu \mathrm{g})$ was loaded for SDS-polyacrylamide gel electrophoresis and transferred to nitrocellulose membranes. Membranes were blocked with $5 \%$ nonfat milk for $1 \mathrm{~h}$. Membranes were incubated with specific primary antibodies against GFAP (1:5000, ab10062, Abcam), neurocan (1:500, ab26003, Abcam), phosphacan (1:1000, P8874, Sigma), Rab7 (1:2000, ab50533, Abcam), Lamp1 (1:500, ab24170, Abcam) and cathepsin B (1:500, 06-480, Millipore), and $\beta$-actin (1:5000, A5441, Sigma) overnight at $4{ }^{\circ} \mathrm{C}$. Corresponding secondary antibodies conjugated with $\mathrm{HRP}$ anti-mouse $\operatorname{lgG}(\mathrm{H}+\mathrm{L})$ (1:10,000, 042-06-18-06, KPL) or anti-rabbit lgG $(\mathrm{H}+\mathrm{L})(1: 10,000$, 042-06-15-06, KPL) were applied for $1 \mathrm{~h}$ at room temperature. Immunoreactivity was detected with an Odyssey scanner (LI-COR). Blots were captured by an Odyssey scanner (LI-COR). Densitometric analyses of the bands were quantitatively analyzed with ImageJ software. $\beta$-actin (1:5000, A5441, Sigma) served as the loading control.

\section{Measurement of lysosomal stability}

The acridine orange (AO) (318337, Sigma-Aldrich) staining assay is widely used for evaluations of LMP. AO is a lysosomotropic base and a metachromatic fluorophore. AO captures protons inside the acidic vacuolar compartment, preferentially in secondary lysosomes, and retains its charged form, which results in red fluorescence. AO relocates to the cytosol from the lysosomes when LMP is increased, which produces a cytoplasmic diffuse green fluorescence and reduced red fluorescence. Cells were subjected to OGD treatment for $6 \mathrm{~h}$ and reperfusion for $24 \mathrm{~h}$ and then incubated with $5 \mu \mathrm{g} / \mathrm{mL}$ of $A O$ in complete medium for $15 \mathrm{~min}$ at $37^{\circ} \mathrm{C}$. Images were obtained by a confocal microscope (LSM 710; Carl Zeiss Co. Ltd., Oberkochen, Germany).

\section{Statistical analysis}

The data are presented as the mean $\pm S D$. Statistical analyses were performed using analysis of variance (ANOVA) for multiple comparisons with Tukey's post-hoc test, and the difference between two groups was evaluated using Student's $t$-test. Ordinal values were analyzed using analysis of variance (ANOVA) and Bonferroni's post hoc correction. $P<0.05$ was considered statistically significant.

\section{RESULTS}

Rab7 was increased in astrogliosis and glial scar formation following ischemia in vivo and in vitro

Astrocytes in the ischemic brain undergo important morphological modifications, such as hyperplasia and hypertrophy, in the peri-infarct region. These astrocytes, also referred to as reactive astrogliosis, subsequently develop into the glial scar [31, 32]. 
Protein levels of GFAP, phosphacan and neurocan are increased in the scar [33]. We measured GFAP and phosphacan protein levels in the peri-infarct region 2, 4, $6 \mathrm{~h}, 1,3,7$, and 14 days after tMCAO with Western blot analysis. The results revealed no significant differences in GFAP (Fig. 1a) or phosphacan (Fig. 1a) levels between the sham group and tMCAO groups at 2,4 , or $6 \mathrm{~h}$. GFAP $(P=0.0018)$ and phosphacan $(P=0.0070)$ protein levels increased significantly from 1 to 7 days after focal ischemia. Protein levels of phosphacan $(P=0.0111)$ exhibited a slight decrease 14 days after tMCAO.
Rab7 may contribute to cerebral tMCAO injuries [34]. However, whether Rab7 participates in astrogliosis and glial scar formation following tMCAO is not known. Our results revealed an increase in Rab7 levels on day $1(P=0.0141)$, which peaked at day $7(P=0.0090)$ and decreased at day $14(P=0.0477)$ in the peri-infarct region of cerebral cortex after tMCAO injury (Fig. 1a). Immunohistochemistry revealed a robust increase in Rab7 $(P=0.0013) 7$ days after tMCAO injury (Fig. $1 \mathrm{~b})$.

We established OGD6 h/Re12 $\mathrm{h}$ and OGD6 h/Re24 h glial scar formation models in primary cultured astrocytes to mimic the a

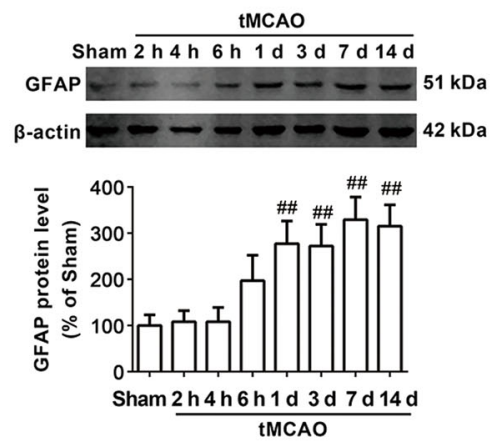

b
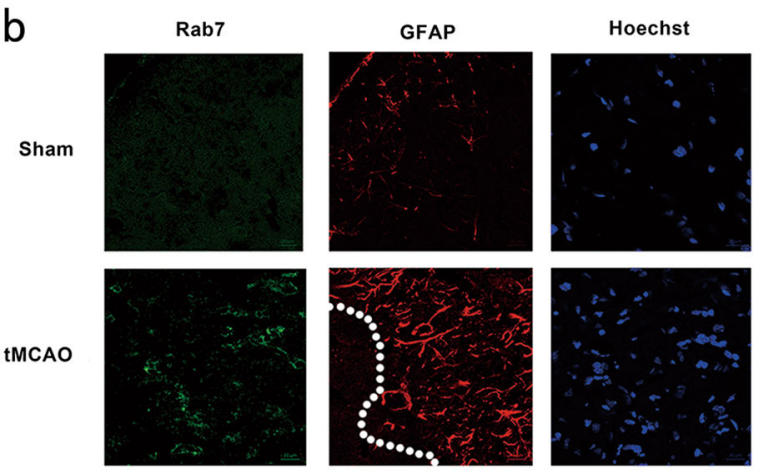
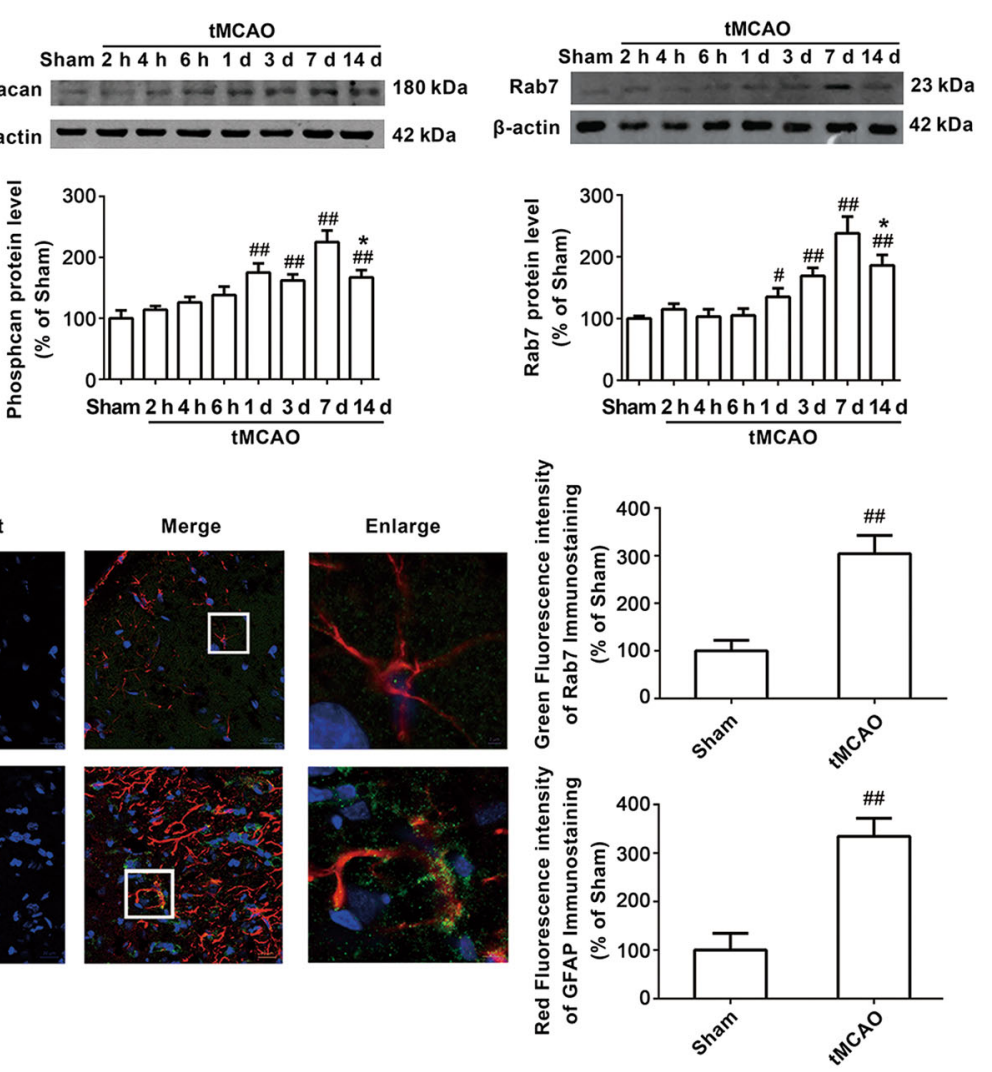

C
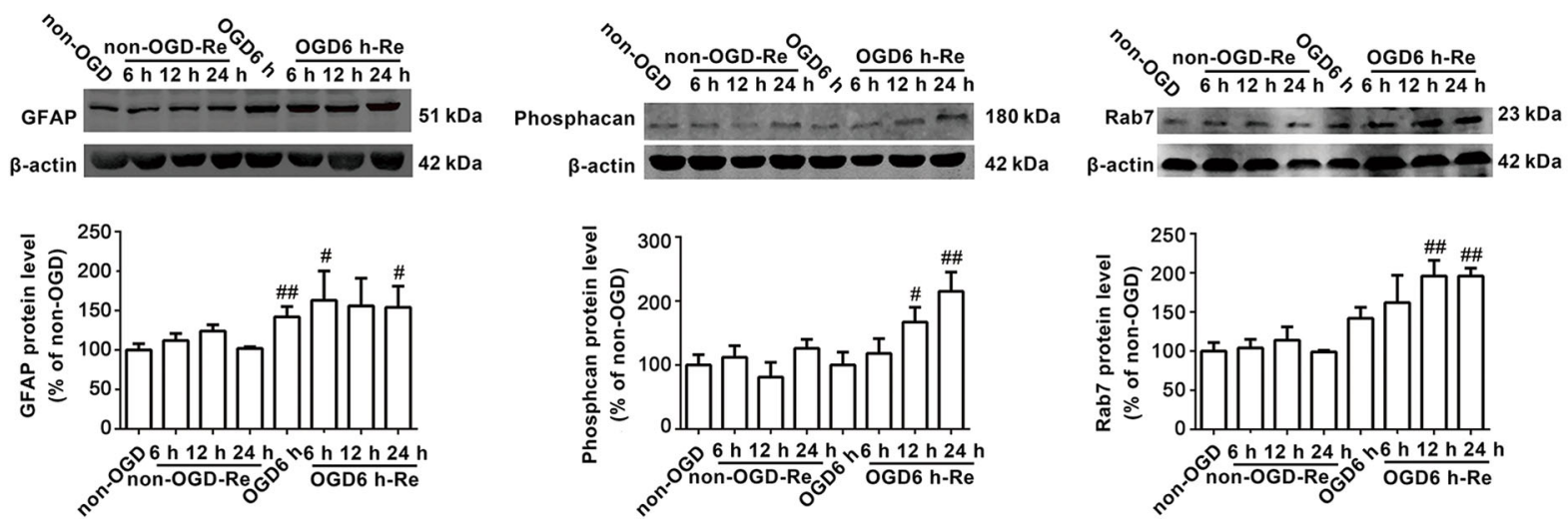

Fig. 1 The time-course changes of GFAP, Phosphacan and Rab7 protein. a Representative images of GFAP, Phosphacan and Rab7 in the periinfarct zones of the sham or cerebral ischemic cortex at 2, 4, $6 \mathrm{~h}, 1,3,7$, and 14 days after reperfusion following tMCAO for 90 min with Western blotting analysis. Columns represent quantitative analysis of immunoblots respectively. $\beta$-actin protein was used as a loading control. Mean \pm SD. $n=3$. ${ }^{\# \#} P<0.01$ vs. sham-operated group (sham); ${ }^{*} P<0.05$ vs. tMCAO for 7 days group. b Representative images of Rab7, GFAP, and Hoechst staining in the peri-infarct zones of the sham or cerebral ischemic cortex at 7 days after reperfusion following tMCAO for 90 min (Rab7: green; GFAP: red; Hoechst: blue). Quantification of green fluorescence intensity of Rab7 immunostaining. Quantification of red fluorescence intensity of GFAP immunostaining. Mean \pm SD. $n=6$. ${ }^{\# \# ~} P<0.01$ vs. sham group. c Representative images of GFAP, Phosphacan and Rab7 at OGD $6 \mathrm{~h}$ and reoxygenaration for 6,12 , and $24 \mathrm{~h}$ with Western blotting analysis. Columns represent quantitative analysis of immunoblots respectively. $\beta$-actin protein was used as a loading control. Mean \pm SD. $n=3 .{ }^{\#} P<0.05,{ }^{\# \#} P<0.01$ vs. non-OGD group. Statistical analysis was carried out with a one-way ANOVA followed by a post-hoc Tukey test 

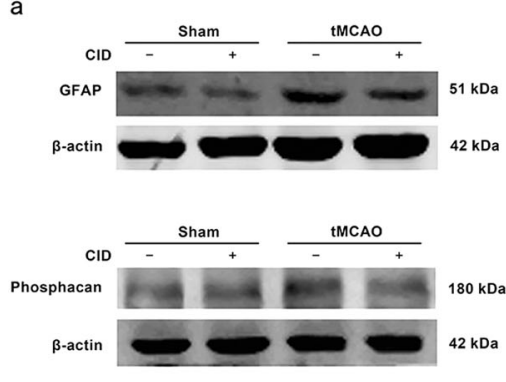

C
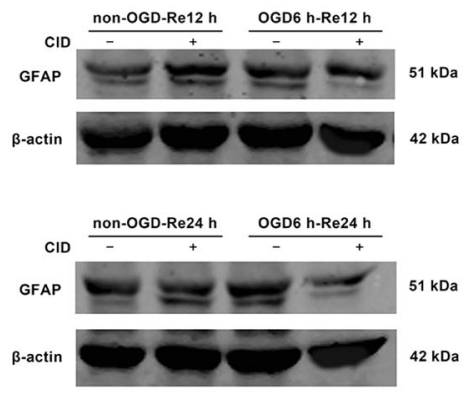
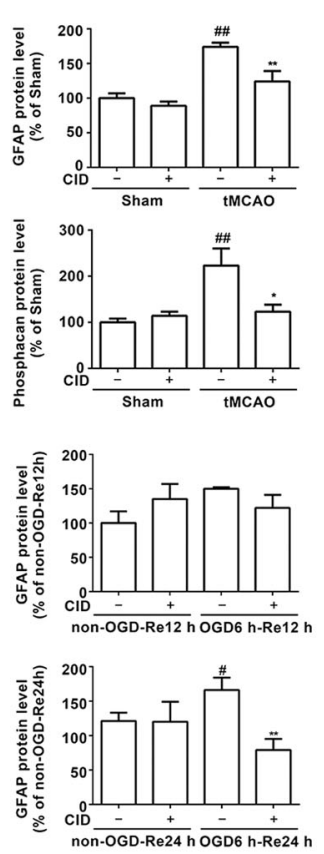
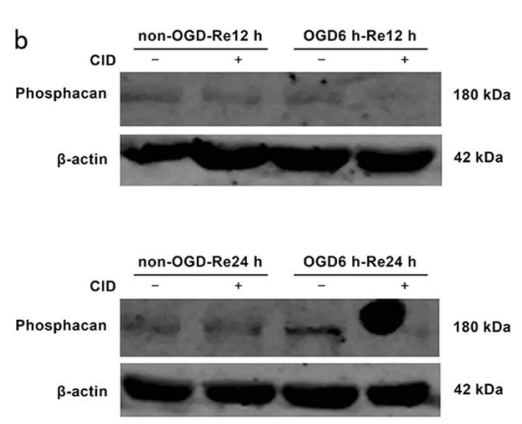

d
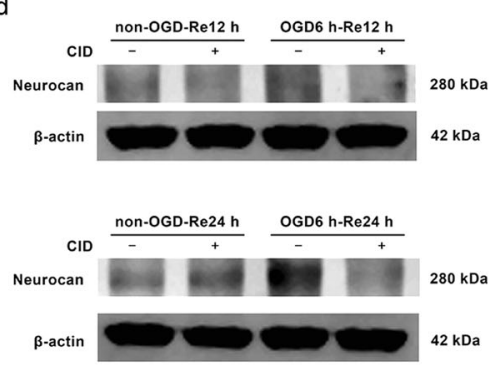
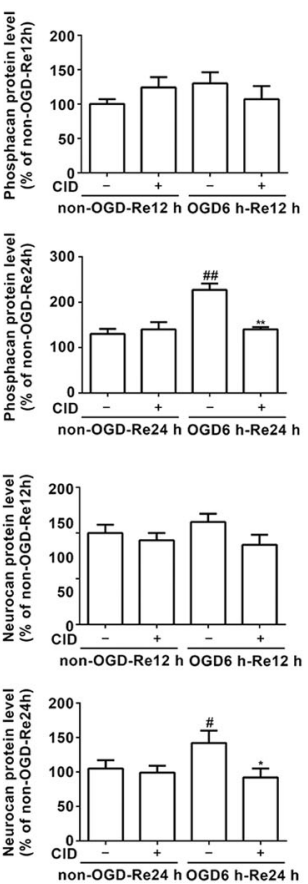

Fig. 2 Rab7 receptor antagonist inhibits the expression of GFAP, Phosphacan and Neurocan after OGD/Re and tMCAO. a Representative images of GFAP and Phosphacan in the peri-infarct zones of the sham or cerebral ischemic cortex at 7 days after reperfusion following tMCAO for $90 \mathrm{~min}$ with Western blotting analysis. Columns represent quantitative analysis of immunoblots respectively. $\beta$-actin protein was used as a loading control. Mean \pm SD. $n=3$. ${ }^{\# \#} P<0.01$ vs. sham group. ${ }^{*} P<0.05,{ }^{* *} P<0.01$ vs tMCAO for 7 days group. $\mathbf{b}$, $\mathbf{c}$, $\mathbf{d}$ Representative images of Phosphacan, GFAP and Neurocan at OGD $6 \mathrm{~h}$ and reoxygenaration for 12 and $24 \mathrm{~h}$ with Western blotting analysis. Columns represent quantitative analysis of immunoblots respectively. $\beta$-actin protein was used as a loading control. Mean \pm SD. $n=3$. ${ }^{\#} P<0.05$, \#\# $P<0.01$ vs. nonOGD-Re24h group. ${ }^{*} P<0.05,{ }^{* *} P<0.01$ vs. OGD6h-Re24h group. Statistical analysis was carried out with a one-way ANOVA followed by a post hoc Tukey test

tMCAO injury in vitro using a previous described method [12]. The results demonstrated that OGD6 $\mathrm{h} / \mathrm{Re}$ induced an increase in GFAP $(P=0.0080)$ (Fig. 1c) and phosphacan $(P=0.0096)$ (Fig. 1c) protein levels that peaked at OGD6 $\mathrm{h} / \mathrm{Re} 24 \mathrm{~h}$. Rab7 protein levels increased at OGD6 h/Re12 h $(P=0.0019)$ and peaked at OGD6 h/ Re24h $(P=0.0004)$ in reactive astrocytes (Fig. 1c), which is consistent with the in vivo results. No differences in Rab7 levels were observed between the non-OGD group, non-OGD/Re group, OGD6 $\mathrm{h}$ group or the OGD6 $\mathrm{h} / \mathrm{Re} 6 \mathrm{~h}$ group.

These results indicate that Rab7 protein levels in the glial scar positively correlated with reactive astrogliosis and glial scar formation after ischemic stroke injury in vivo and in vitro.

Rab7 receptor antagonist attenuates ischemic stroke-induced astrogliosis in vivo and in vitro

Numerous astrocytes are activated after reperfusion, and excessive astrogliosis further extends processes toward the ischemic core. The glial scar matures in the peri-infarct region, which demarcates the ischemic core from healthy tissue [35-38]. This scar inhibits axonal sprouting and limits regeneration [13]. However, the role of Rab7 in astrogliosis and glial scar formation in ischemic stroke remains unknown. The Rab7 receptor antagonist CID reduces Rab7-GTP levels [39]. In the current study, we found that CID markedly decreased GFAP ( $P=0.0058$ ) (Fig. 2a) and phosphacan $(P=0.0123)$ (Fig. 2a) protein levels in the peri-infarct region 7 days after tMCAO injury.

The Rab7 receptor antagonist reduced phosphacan $(P=0.005)$ (Fig. 2b), GFAP $(P=0.0033)$ (Fig. $2 \mathrm{c})$, and neurocan $(P=0.0175)$ (Fig. 2d) protein levels at OGD6 $\mathrm{h} / \mathrm{Re} 24 \mathrm{~h}$, which is consistent with the results in vivo. Immunofluorescence analysis revealed that the Rab7 receptor antagonist decreased phosphacan $(P=0.0013)$ (Fig. 3a) and neurocan ( $P=0.0011)$ (Fig. 3b) immunoreactivity in reactive astrocytes at OGD6 $\mathrm{h} / \mathrm{Re} 24 \mathrm{~h}$.
These results suggest that the Rab7 receptor antagonist inhibits astrogliosis and glial scar formation after ischemic brain injury.

Rab7 receptor antagonist reduces brain atrophy and ameliorates behavioral symptoms during the recovery phase of tMCAO Ischemic stroke invariably forms an infarction core that causes severe neurological impairment [6]. Our results demonstrated that a Rab7 receptor antagonist significantly attenuated brain atrophy volumes 28 days $(P<0.0010)$ after tMCAO (Fig. 4a). CID also increased grip strength (Fig. 4b), decreased right forelimb use in the cylinder test (Fig. 4b) and improved neurological impairment scores (Fig. 4b) from 1 to 28 days after tMCAO. These results suggest that a Rab7 receptor antagonist promotes the recovery of brain function during the late stage of brain injury after tMCAO.

Rab7 receptor antagonist protects against OGD/Re-induced astrocytic cell injury

We next examined whether a Rab7 receptor antagonist exhibited protective effects on astrocytes after OGD/Re in vitro. The present study established OGD6 h/Re12 $\mathrm{h}$ and OGD6 h/Re24 h-induced glial scar formation models in primary cultured astrocytes. We detected the LDH leakage rate as an index of astrocytic cell death. We found that $80 \mathrm{nM}$ CID significantly protected against astrocytic cell injury and reduced the LDH leakage of astrocytes after OGD6 h/Re12 h $(P=0.0151)$ (Fig. 5a) and OGD6 h/Re24 h $(P=0.0128)$ (Fig. 5b). These results demonstrate that a Rab7 receptor antagonist directly protects astrocytes against OGD/ Re-induced injury.

Rab7 receptor antagonist inhibits the colocalization of Rab7, cathepsin B, and Lamp1 after OGD/Re and tMCAO injury Cathepsin $B$ is transported from late endosomes to lysosomes after transient cerebral ischemia [40], and excessive cathepsin B 

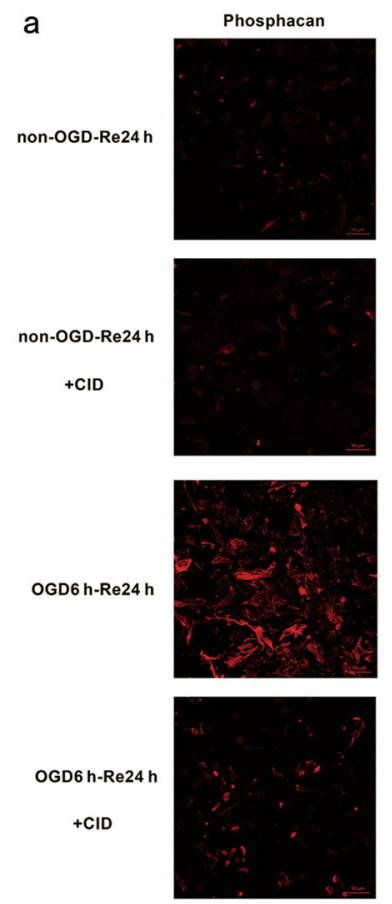

b
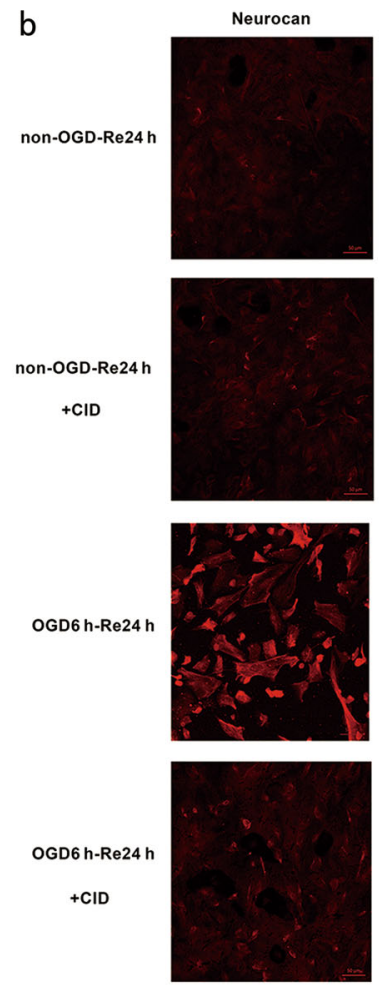

GFAP
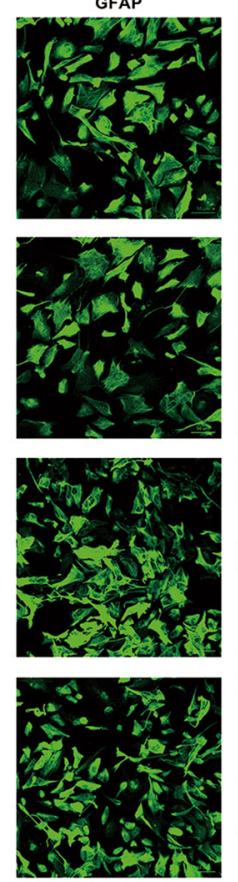

GFAP
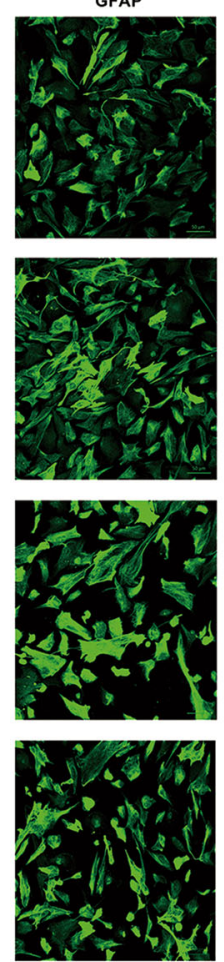
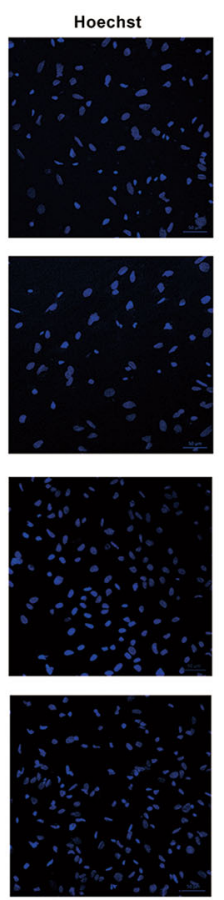

Hoechst
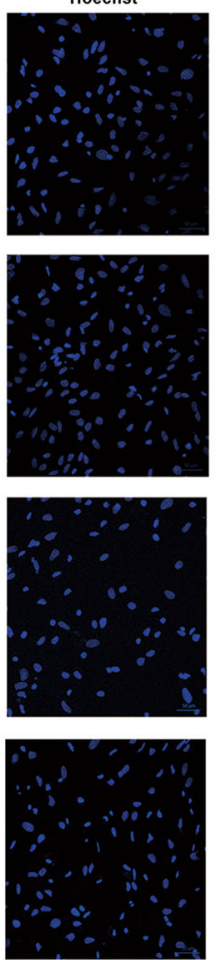

Merge
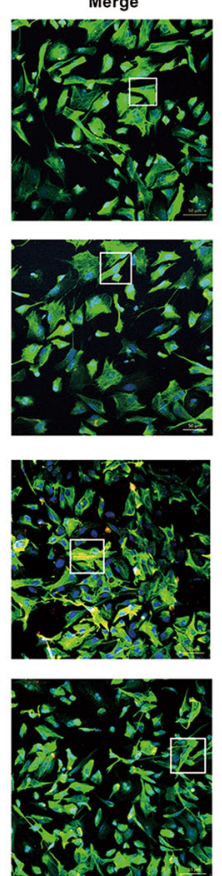

Merge
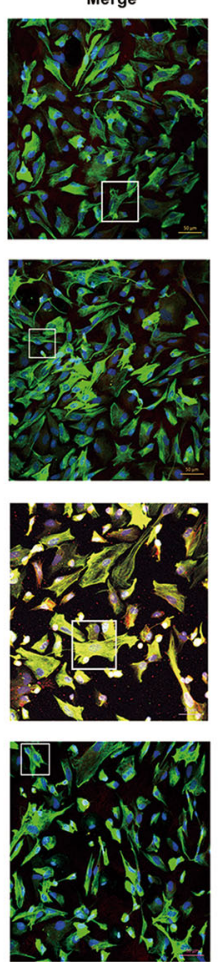
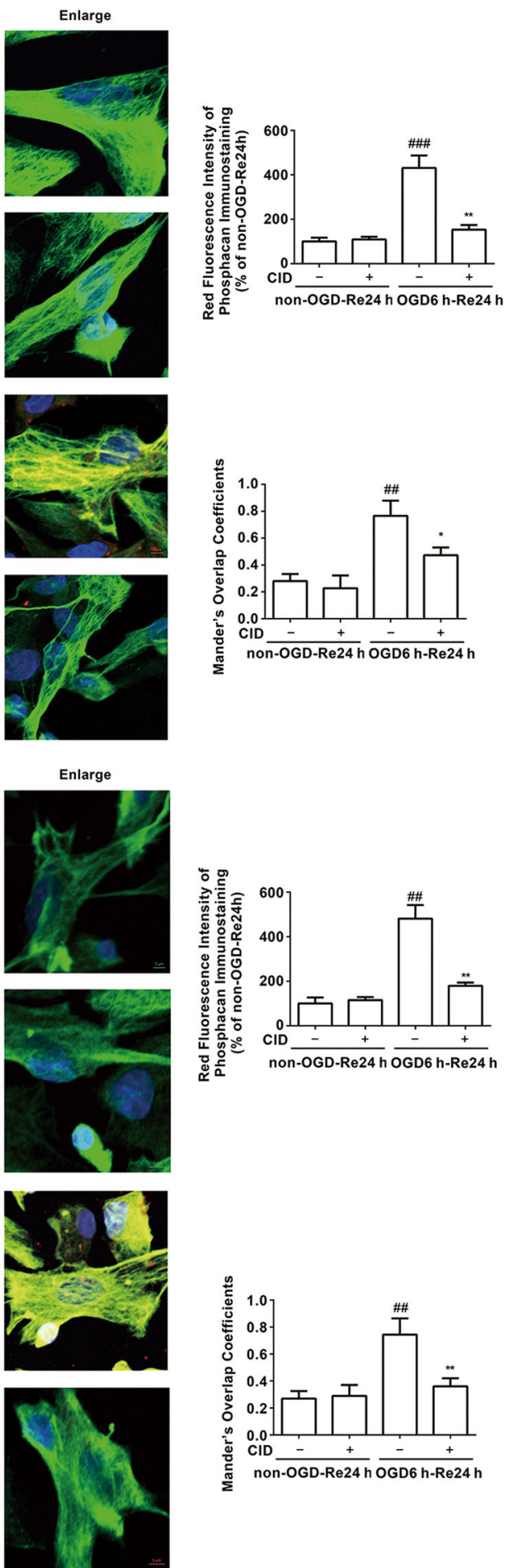

Fig. 3 Rab7 receptor antagonist inhibits the immunoreactivity of Phosphacan and Neurocan after OGD/Re-24h. a Representative images of Phosphacan, GFAP, and Hoechst staining in astrocytes after OGD for $6 \mathrm{~h}$ and reoxygenaration for $24 \mathrm{~h}$ (Phosphacan: red; GFAP: green; Hoechst: blue). Quantification of red fluorescence intensity of Phosphacan immunostaining. Mander's overlap coefficients demonstrated the co-localization between phosphacan and GFAP. Mean \pm SD. $n=6 .{ }^{\# \#} P<0.01$ vs non-OGD-Re24h group; ${ }^{* *} P<0.01$, ${ }^{*} P<0.05$ vs. OGD6h-Re24h group. b Representative images of Neurocan, GFAP, and Hoechst staining in astrocytes after OGD for $6 \mathrm{~h}$ and reoxygenaration for $24 \mathrm{~h}$ (neurocan: red; GFAP: green; Hoechst: blue). Quantification of red fluorescence intensity of Neurocan immunostaining. Mander's overlap coefficients demonstrated the co-localization between Neurocan and GFAP. Mean \pm SD. $n=6$. ${ }^{\# \#} P<0.01$ vs. non-OGD-Re24 h group; ${ }^{* *} P<0.01$ vs. OGD6h-Re24h group. Statistical analysis was carried out with a one-way ANOVA followed by a post-hoc Tukey test 
a

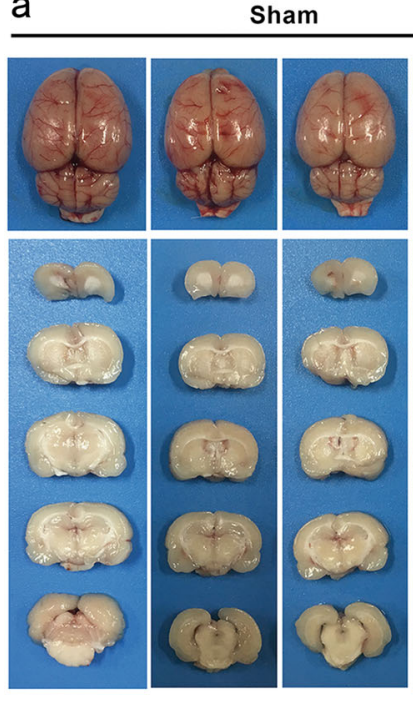

tMCAO
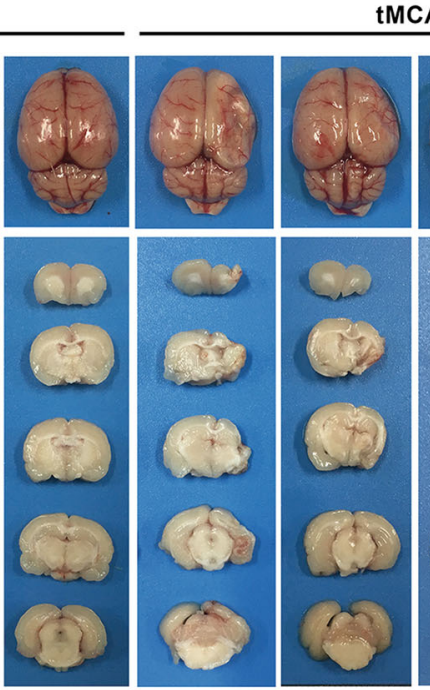
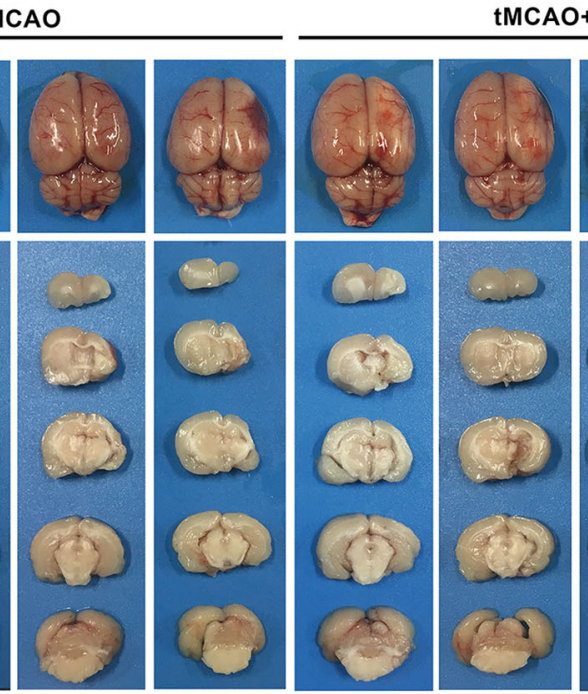

MCAO+CID
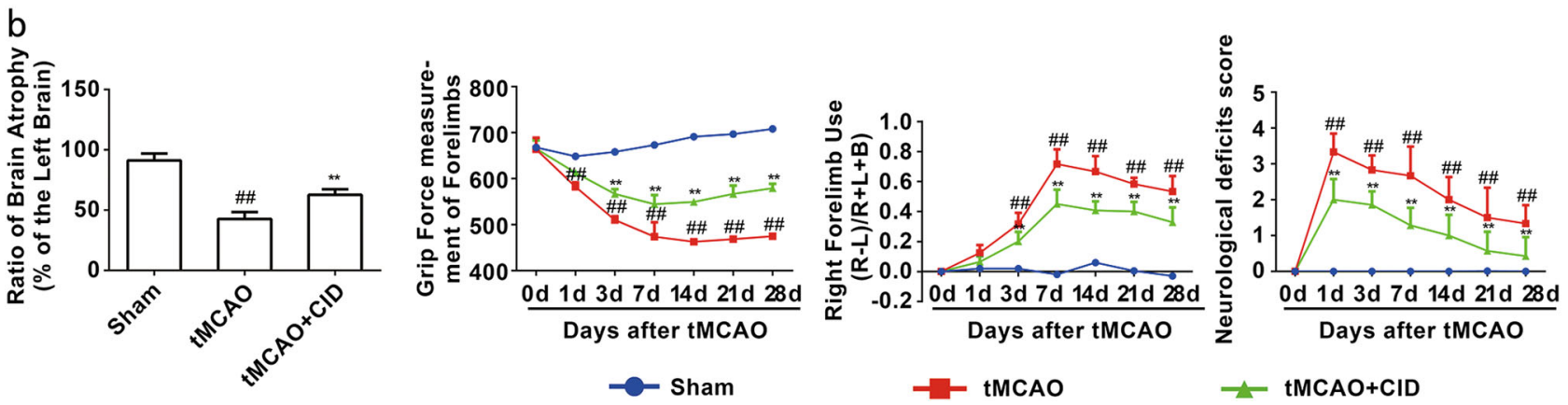

Fig. 4 Rab7 receptor antagonist promotes brain functional recovery in rats. a Rab7 receptor antagonist reduces brain atrophy at 28 days after reperfusion following tMCAO for $90 \mathrm{~min}$. Quantitative analysis of the residual right brain volume/left brain volume. $\mathbf{b}$ The grip strength test; The cylinder test; The 6-point scale neurological score test. Mean \pm SD. $n=10$. ${ }^{\# \#} P<0.01$ vs. sham group; ${ }^{* *} P<0.01$ vs. tMCAO group. Statistical analysis was carried out with a two-way ANOVA followed by a post-hoc Tukey test for multiple comparisons and Student's $t$-test for two groups. Ordinal values were analyzed by two-way analysis of variance (ANOVA) using Bonferroni's post-hoc correction
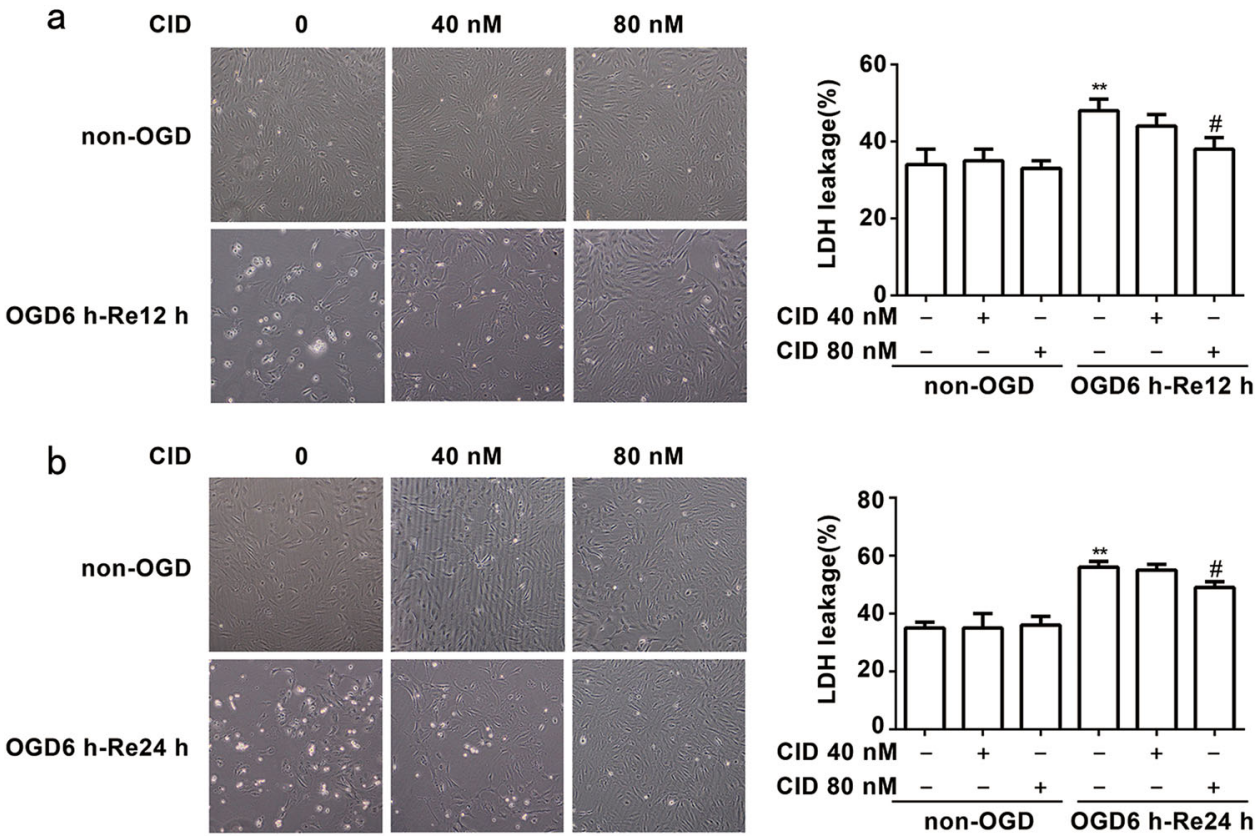

Fig. 5 Rab7 receptor antagonist protects against OGD/Re-induced astrocytic cell injury. a, b Representative light microscope images of astrocytes at OGD for $6 \mathrm{~h}$ and reoxygenaration for 12 and $24 \mathrm{~h}$. Columns represent quantitative analysis of LDH leakage respectively. Mean \pm SD. $n=6$. ${ }^{* *} P<0.01$ vs. non-OGD group; ${ }^{\#} P<0.05$ vs. OGD6h-Re12 h or OGD6h-Re24 h group. Statistical analysis was carried out with a oneway ANOVA followed by a post-hoc Tukey test 
a N-OGD-Re24 + CID
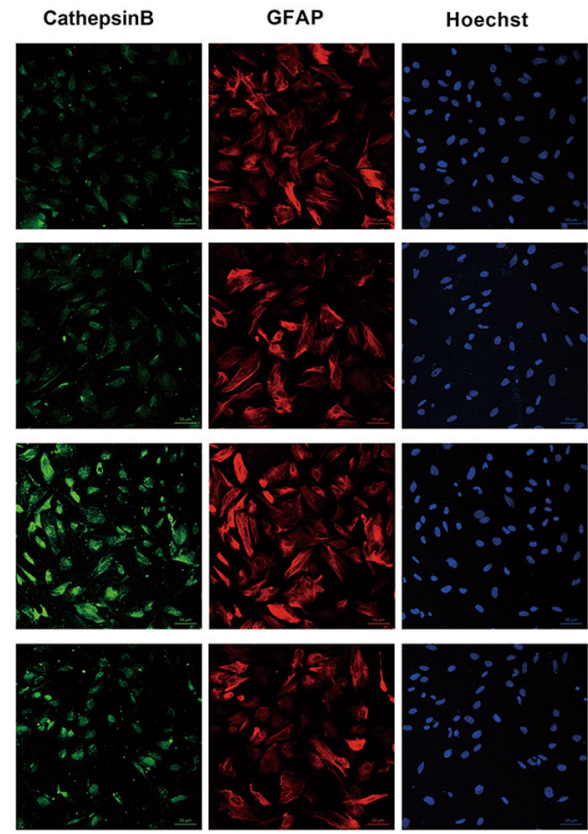

b

non-OGD-Re24
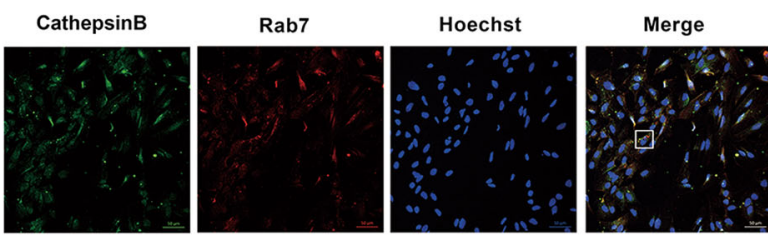

non-OGD-Re24

+ CID
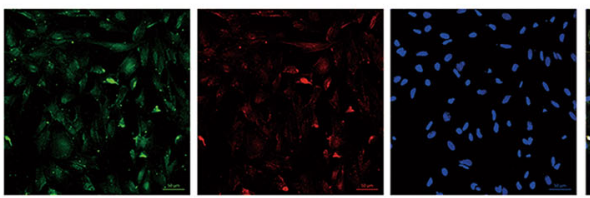

OGD6 h-Re24 h

+ CID
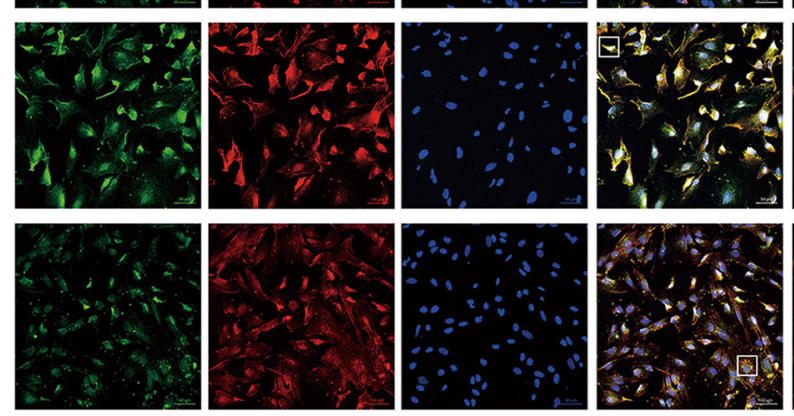
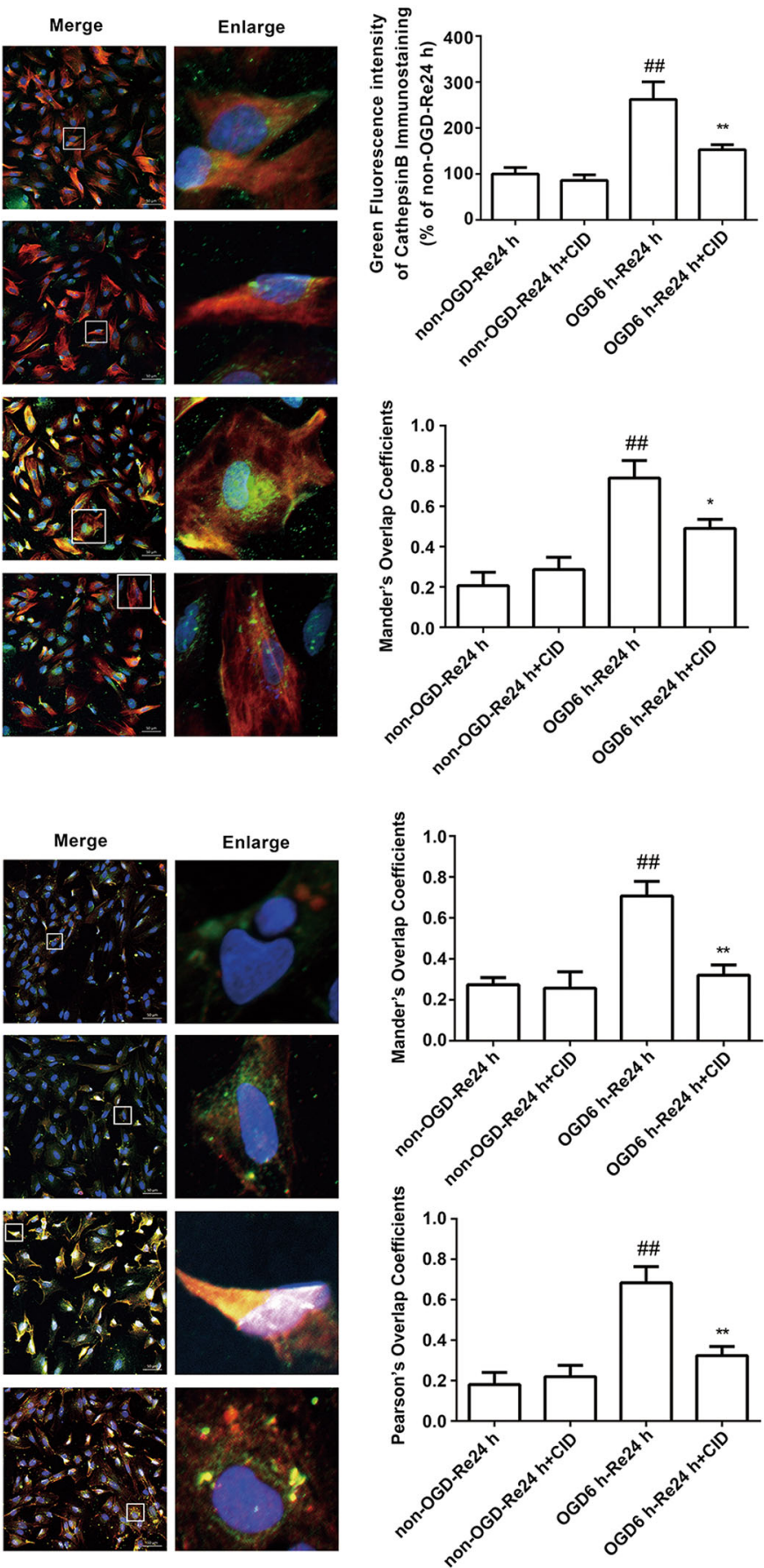

Fig. 6 Rab7 receptor antagonist inhibits the co-localization of cathepsin B and Rab7. a Representative images of cathepsin B, GFAP, and Hoechst staining in astrocytes after OGD for $6 \mathrm{~h}$ and reoxygenaration for $24 \mathrm{~h}$ (cathepsin B: green; GFAP: red; Hoechst: blue).Quantification of green fluorescence intensity of cathepsin B immunostaining. Mander's overlap coefficients demonstrated the co-localization between cathepsin B and GFAP. Mean \pm SD. $n=6$. ${ }^{\# \#} P<0.01$ vs. non-OGD-Re24 h group; ${ }^{*} P<0.05,{ }^{* *} P<0.01$ vs. OGD6 h-Re24 h group. b Representative images of cathepsin B, Rab7, and Hoechst staining in astrocytes after OGD for $6 \mathrm{~h}$ and reoxygenaration for $24 \mathrm{~h}$ (cathepsin B: green; Rab7: red; Hoechst: blue). Mander's overlap coefficients and Pearson's overlap coefficients demonstrated the co-localization between cathepsin B and Rab7. Mean \pm SD. $n=6$. ${ }^{\# \#} P<0.01$ vs non-OGD-Re24 h group; ${ }^{* *} P<0.01$ vs. OGD6h-Re24h group. Statistical analysis was carried out with a oneway ANOVA followed by a post hoc Tukey test

activation in lysosomes decreases the stability of the lysosome membrane after ischemic injury [6]. Therefore, we examined whether CID treatment inhibited excessive cathepsin B transport to lysosomes to indirectly stabilize the lysosomal membrane and play a protective role in astrocytes after OGD/Re and tMCAO injury. Immunofluorescence and immunohistochemistry revealed that CID treatment inhibited the colocalization of Rab7 and cathepsin B $(P=0.0016)$ (Fig. 6a, b) and Rab7 and Lamp1 after tMCAO $(P=0.0037)$ (Fig. 7a) and OGD/Re $(P=0.0016)$ (Fig. 7b). These results suggest that CID blocks excessive cathepsin $B$ transport to lysosomes, which protects the stability of lysosomal membranes. 
a

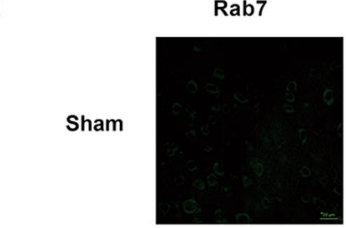

Sham+CID

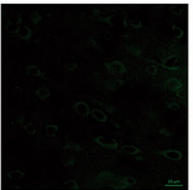

IMCAO
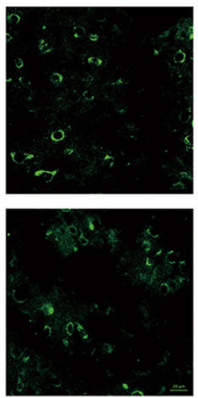

b

tMCAO+CID

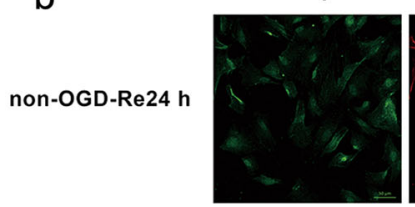

non-OGD-Re24 h

$+C I D$
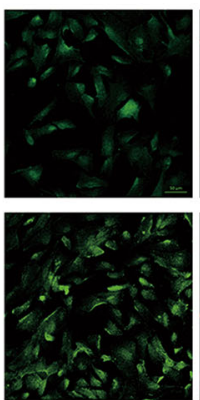

OGD6 h-Re24 h

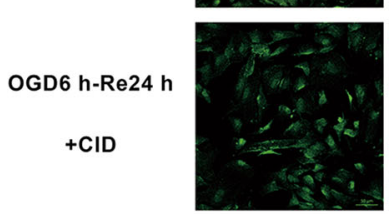

Lamp1
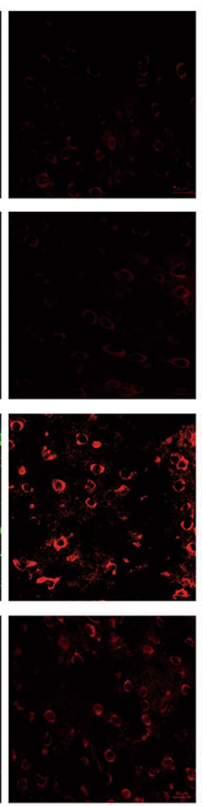

Rab7
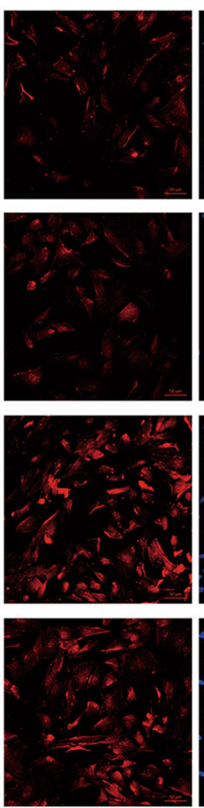

Hoechst
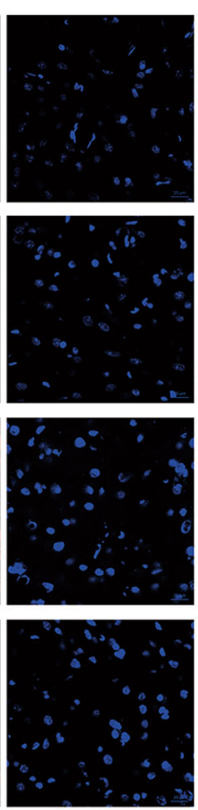

Hoechst
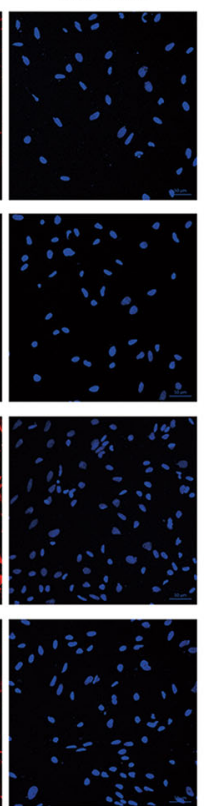

Merge
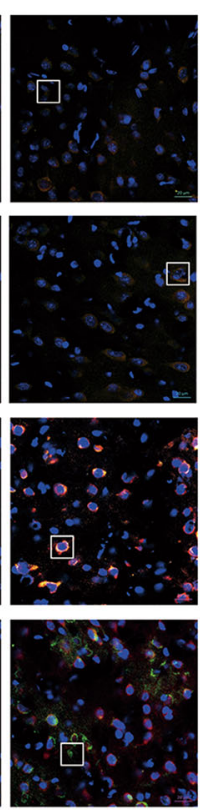

Merge
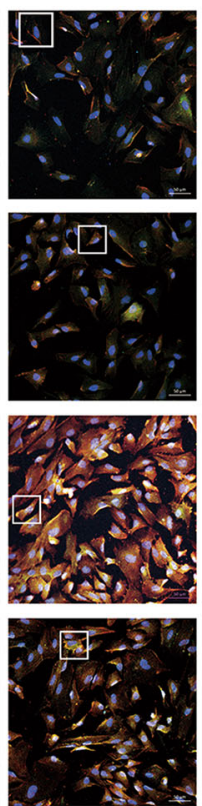

Enlarge
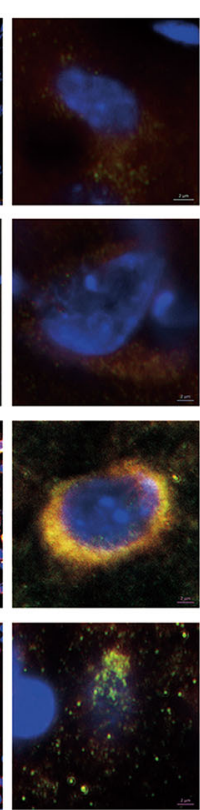

Enlarge
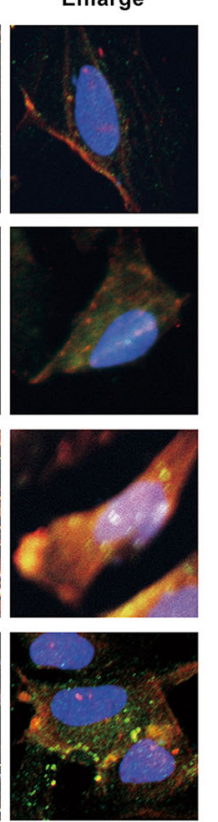
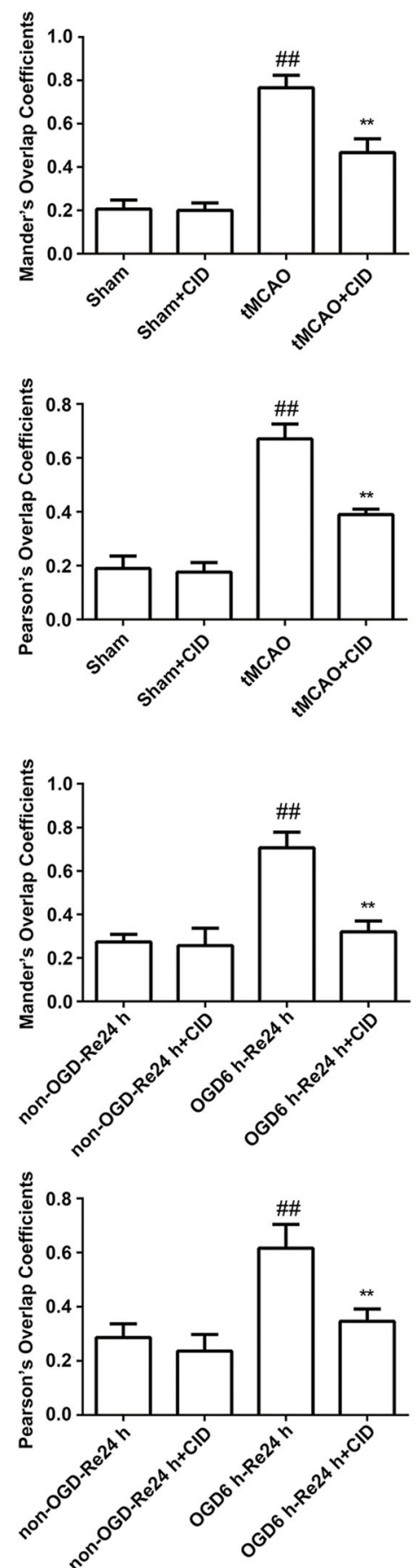

Fig. 7 Rab7 receptor antagonist inhibits the co-localization of Rab7 and Lamp1 after tMCAO injury and OGD/Re. a Representative images of Rab7, Lamp1, and Hoechst staining in the peri-infarct zones of the sham or cerebral ischemic cortex at 7 days after reperfusion following tMCAO for 90 min (Rab7: green; Lamp1: red; Hoechst: blue). Mander's overlap coefficients and Pearson's overlap coefficients demonstrated the co-localization between Rab7 and Lamp1. Mean \pm SD. $n=6$. ${ }^{\# \#} P<0.01$ vs. sham group; ${ }^{* *} P<0.01$ vs. 7 days of tMCAO group. b Representative images of Lamp1, Rab7, and Hoechst staining in astrocytes after OGD for $6 \mathrm{~h}$ and reoxygenaration for 24 h (Lamp1: green; Rab7: red; Hoechst: blue). Mander's overlap coefficients and Pearson's overlap coefficients demonstrated the co-localization between Lamp1 and Rab7. Mean \pm SD. $n=6$. ${ }^{\# \#} P<0.01$ vs. non-OGD-Re24 h group; ${ }^{* *} P<0.01$ vs. OGD6h-Re24 h group. Statistical analysis was carried out with a one-way ANOVA followed by a post-hoc Tukey test

Rab7 receptor antagonist blocks cathepsin B activation and release from lysosomes into the cytoplasm

Yuan and colleagues reported that transient cerebral ischemia induced a massive buildup of damaged late endosomes, which resulted in a cascade of events that led to the fatal release of cathepsin $\mathrm{B}$ and delayed neuronal death after transient cerebral ischemia $[26,40]$. Our previous data demonstrated that inhibition of cathepsin B activation and release from lysosomes into the cytoplasm inhibited astrogliosis and glial scar formation after ischemic stroke [6]. Late endosomes transport cathepsins from the Golgi to lysosomes for degradation [40]. Therefore, we hypothesized that the Rab7 receptor antagonist-mediated 
a
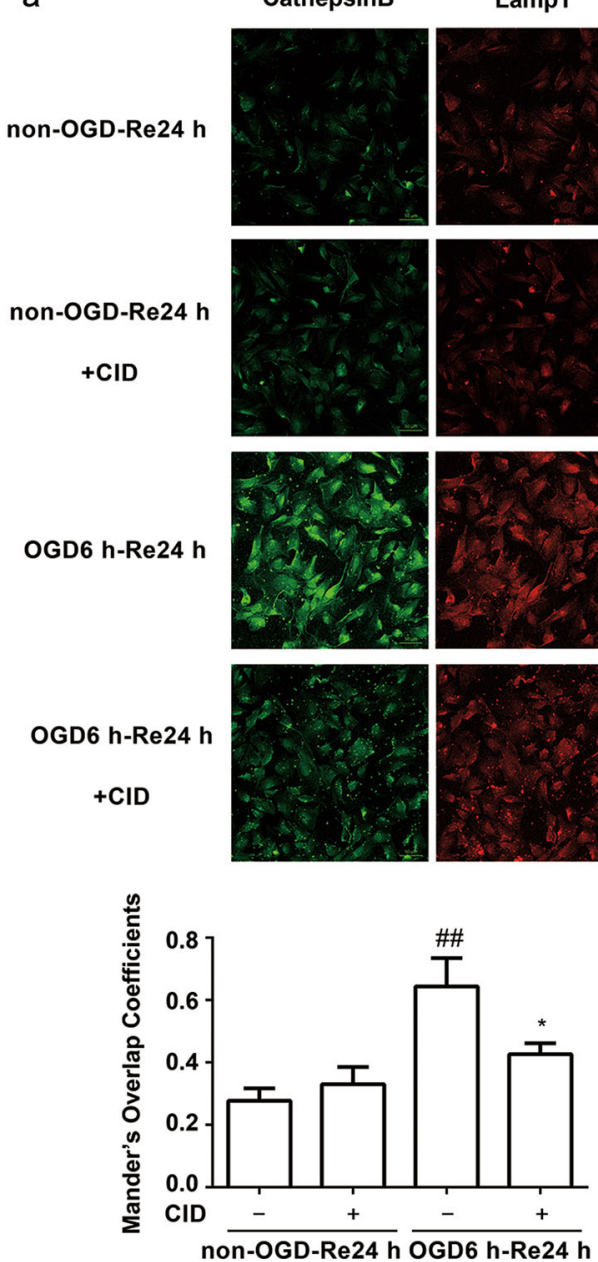

b

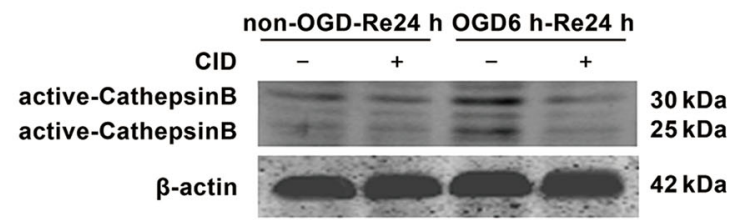

Lamp1
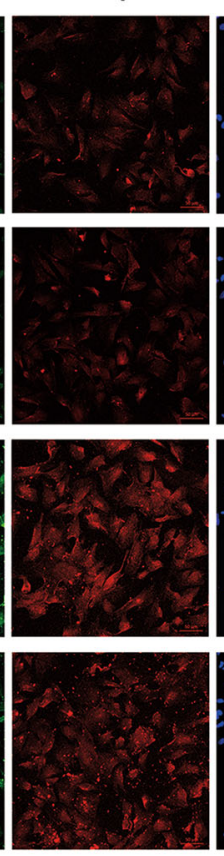

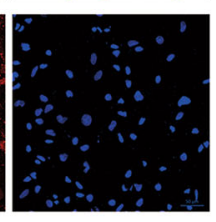

\begin{abstract}
Hoechst
\end{abstract}
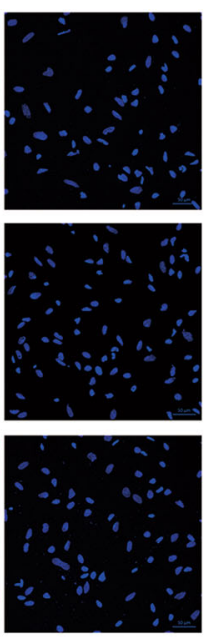

Merge
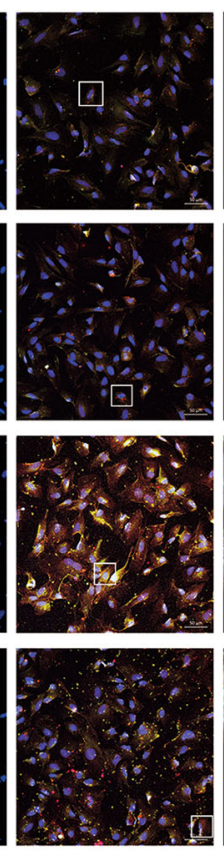

Enlarge
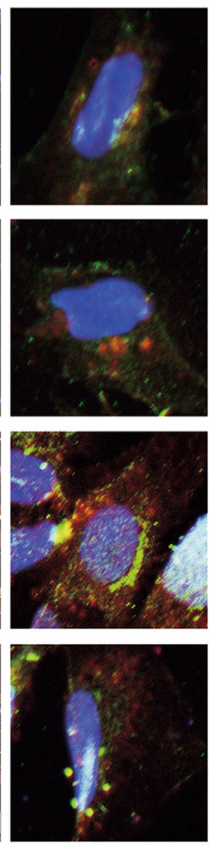
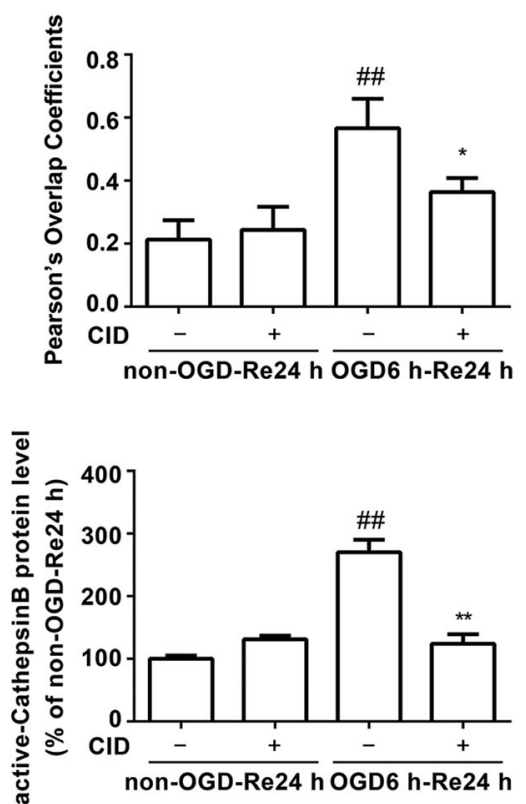

Fig. 8 Rab7 receptor antagonist inhibits release of cathepsin B from the lysosomes into the cytoplasm after OGD/Re, and inhibits the activation of cathepsin B after OGD/Re. a Representative images of cathepsin B, Lamp1, and Hoechst staining in astrocytes after OGD for $6 \mathrm{~h}$ and reoxygenaration for $24 \mathrm{~h}$ (cathepsin B: green; Lamp1: red; Hoechst: blue). Mander's overlap coefficients and Pearson's overlap coefficients demonstrated the co-localization between cathepsin B and Lamp1. Mean \pm SD. $n=6 .{ }^{\# \#} P<0.01$ vs. non-OGD-Re24 h group; ${ }^{*} P<0.05$ vs. OGD6h-Re24h group. b Image of active cathepsin B at OGD $6 \mathrm{~h}$ and reoxygenaration for $24 \mathrm{~h}$ with Western blotting analysis. Columns represent quantitative analysis of immunoblots. $\beta$-actin protein was used as a loading control. Mean \pm SD. $n=3$. ${ }^{\# \#} P<0.01$ vs. non-OGD-Re24h group; ${ }^{* *} P<0.01$ vs. OGD6h-Re24h group. Statistical analysis was carried out with a one-way ANOVA followed by a post-hoc Tukey test

inhibition of glial scar formation would be associated with the inhibition of cathepsin $B$ activation and release from lysosomes into the cytoplasm. We examined the effects of CID on OGD/Re-induced cathepsin B activation and release from lysosomes into the cytoplasm in astrocytes using immunofluorescence and Western blot analysis. Fewer fine, granular, perinuclear cathepsin B immunostaining colocalized with Lamp1-positive lysosomes in non-OGD astrocytes (Fig. 8a). This finding is consistent with the predominantly lysosomal location of these proteases [22, 41-44]. Cathepsin B granules became larger and 

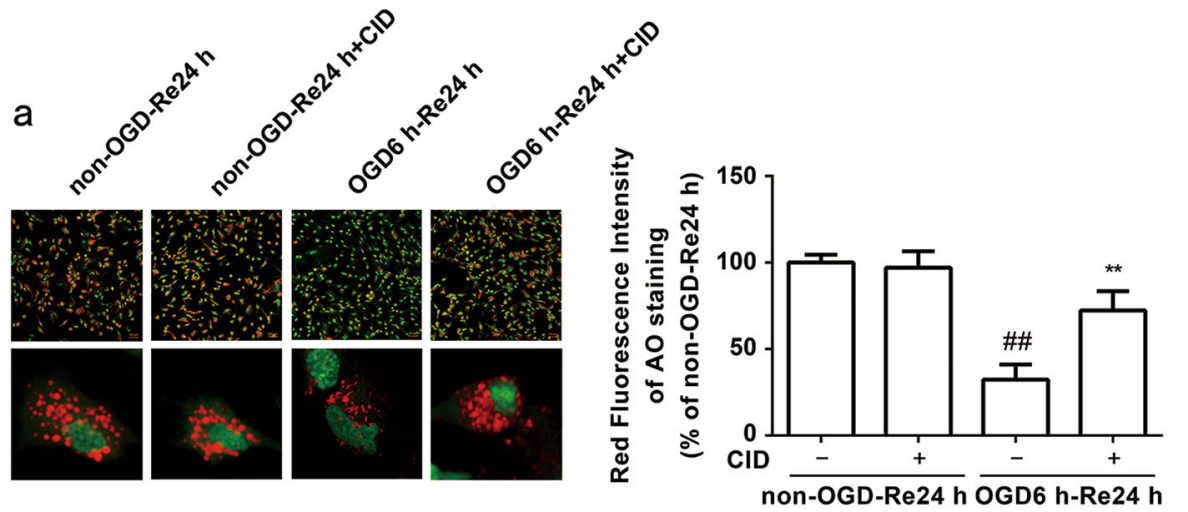

b

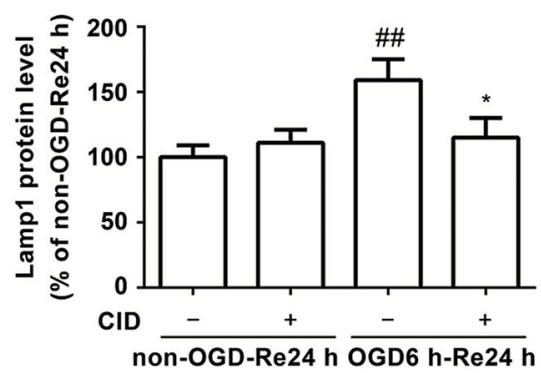

Fig. 9 Rab7 receptor antagonist decreases the lysosomal membrane permeability induced by OGD/Re. a Representative photomicrographs of AO staining images. Quantification of red fluorescence intensity of AO staining. Mean $\pm \mathrm{SD}$. $n=6$. ${ }^{\#} P<0.01$ vs. non-OGD-Re24 h group; ${ }^{* *} P<0.01$ vs. OGD6h-Re24h group. b Image of Lamp1 at OGD $6 \mathrm{~h}$ and reoxygenaration for $24 \mathrm{~h}$ with Western blotting analysis. Columns represent quantitative analysis of immunoblots. $\beta$-actin protein was used as a loading control. Mean \pm SD. $n=3$. ${ }^{\# \#} P<0.01$ vs. non-OGD-Re24h group; ${ }^{*} P<0.05$ vs. OGD6h-Re24h group. Statistical analysis was carried out with a one-way ANOVA followed by a post-hoc Tukey test

inhibition of cathepsin B activation and release from lysosomes into the cytoplasm.

Rab7 receptor antagonist stabilizes OGD/Re-induced lysosomal membrane instability in astrocytes

Cerebral ischemia induces LMP [20, 45], and LMP may mediate cathepsin B cytosolic translocation. Therefore, we evaluated LMP formation using Acridine Orange (AO) assays. AO is a metachromatic fluorophore that is normally cloistered inside lysosomes, which exhibit high levels of red fluorescence and low levels of green fluorescence. AO relocates from lysosomes to the cytosol when disrupted, which manifests as a reduction in red fluorescence and an increase in green fluorescence [45, 46]. OGD/Re induced a reduction in red fluorescence in astrocytes, and treatment with CID markedly inhibited the OGD/Re-induced reduction in red granular fluorescence of $A O$ staining $(P=0.008)$ (Fig. 9a). Western blot analysis demonstrated that CID decreased Lamp1 protein levels after OGD/Re injury $(P=0.0255)$ (Fig. 9b). These data suggest that the Rab7 receptor antagonist stabilizes OGD/Re-induced lysosomal membrane instability in astrocytes.

\section{DISCUSSION}

Rab7 is required for astrogliosis after ischemic stroke

Reactive astrogliosis is a pathological hallmark of the central nervous system (CNS) [47]. Reactive astrocytes contribute to beneficial effects via the secretion of neurotrophic substances that protect neurons and astrocytes at early stages $[6,48]$. However, an excessive proliferation of reactive astrocytes leads to glial scar formation, which is definitely harmful to astrocytic and neuronal repair and axonal regeneration during later periods [6, 10, 48, 49]. The present study demonstrated the important roles of Rab7 in ischemic stroke and provides new evidence that a Rab7 receptor antagonist attenuated astrogliosis and glial scar formation in response to ischemic insult. One novel finding of the current study is that the marker of late endosomes Rab7 [34], participates in astrogliosis and glial scar formation after ischemic stroke. Rab7 was significantly elevated in reactive astrocytes in the periinfarct region of the cerebral cortex following tMCAO or OGD/Re, and the time course of Rab7 upregulation positively correlated with astrogliosis and glial scar formation. The Rab7 receptor antagonist attenuated astrogliosis and glial scar formation in response to ischemic insult. The Rab7 receptor antagonist reduced GFAP and phosphacan protein levels in the peri-infarct region 7 days after tMCAO injury, which is a subacute stage. We further demonstrated that a Rab7 receptor antagonist inhibited OGD/Re-induced astrogliosis in vitro, significantly decreased neurocan and phosphacan immunoreactivity in reactive astrocytes, and reduced GFAP, neurocan and phosphacan protein levels in reactive astrocytes after $\mathrm{OGD} / \mathrm{Re}$ injury. These data indicate that Rab7 is required for astrogliosis after ischemic stroke, and a Rab7 receptor antagonist reduces astrogliosis and glial scar formation.

Rab7 signaling in ischemic astrocytes

The present study also demonstrated the protective effects of a Rab7 receptor antagonist on ischemic stroke. We found that a Rab7 receptor antagonist directly protected against OGD/Re-induced astrocytic cell death. We further found that a Rab7 receptor antagonist reduced the volume of brain atrophy and improved brain function after tMCAO injury. These data demonstrate that a Rab7 receptor antagonist promotes the recovery of brain function in ischemic stroke. Our study suggests that Rab7 activity is an important contributing factor for astrocyte dysfunction and reactive astrogliosis, and the targeting of Rab7 is a better strategy to maintain normal astrocytic function, block the excessive reactive astrogliosis, and improve brain function after ischemic stroke.

Association between Rab7 and cathepsin B in astrogliosis after ischemic stroke

Another novel finding of the current study is that the Rab7 receptor antagonist-mediated inhibition of reactive astrocytes is 
associated with the ability of CID to stabilize the astrocytic lysosomal membrane, which blocked cathepsin B activation and release from the lysosomes into the cytoplasm during astrogliosis and glial scar formation after ischemic injury. A previous study reported that Rab7 activation exerted a neuroprotective role in hypoxic preconditioning against transient global cerebral ischemia-induced neuronal injury in adult rats [50]. These results confirmed that the hypoxic preconditioning-induced neuroprotection was not the result of an increase in lysosomes or improvement of lysosomal function after transient global cerebral ischemia. However, another study demonstrated that transient cerebral ischemia produced a massive buildup of Golgi fragments, transport vesicles (TVs), late endosomes (LEs), and the release of a $33 \mathrm{kDa}$ LE type of cathepsin B, followed by delayed neuronal death [38]. Our findings are consistent with these latter findings, which indicated that the roles of Rab7 were associated with cathepsin B after tMCAO injury. A Rab7 receptor antagonist reversed tMCAO and OGD/Re-induced lysosomal swelling and the rupture of reactive astrocytes in the present study. Further experiments revealed that a Rab7 receptor antagonist reduced the aggregates and diffusion of cathepsin B (the cytoplasmic location of cathepsin B) and decreased cathepsin B activity in reactive astrocytes. We also found that a Rab7 receptor antagonist inhibited the colocalization of Rab7 and cathepsin B and Rab7 and Lamp1 after OGD/Re and tMCAO injury, which suggests that CID prevented Rab7 transport of cathepsin B to lysosomes after ischemic stroke. These results also indirectly indicate that CID blocked cathepsin B activation and release from lysosomes into the cytoplasm. One intriguing novel finding in the current study is that a Rab7 receptor antagonist stabilized OGD/Re-induced lysosomal membrane instability and inhibited the OGD/Reinduced activation of cathepsin B in astrocytes, which suggests that Rab7 mediates lysosomal membrane instability in ischemic astrocytes.

In conclusion, we demonstrated for the first time that astrocytic Rab7 plays a critical role in reactive astrogliosis and ischemic astrocytic damage after ischemic stroke in vivo and in vitro. We demonstrated that a Rab7 receptor antagonist reduced brain atrophy, ischemic astrocytic damage and reactive astrocyte formation and improved brain function. These benefits were associated with the inhibition of cathepsin B activation and release from lysosomes into the cytoplasm.

\section{ACKNOWLEDGEMENTS}

This work was supported by grants from the National Natural Science Foundation of China (No 30973510, 81171104, 81473211), the Jiangsu Key Laboratory of Neuropsychiatric Diseases (No BM2013003), the Jiangsu Key Laboratory of Preventive and Translational Medicine for Geriatric Diseases, and a project Funded by the Priority Academic Program Development of Jiangsu Higher Education Institutions (PAPD).

\section{AUTHOR CONTRIBUTIONS}

$\mathrm{H}-\mathrm{IZ}$ designed the research; $\mathrm{YQ}, \mathrm{YH}, \mathrm{Y}-\mathrm{mZ}, \mathrm{ML}, \mathrm{YN}$ and $\mathrm{JL}$ performed the research; $\mathrm{YQ}$ analyzed the data; $\mathrm{YQ}$ wrote the manuscript; and $\mathrm{HZ}$ revised the manuscript.

\section{ADDITIONAL INFORMATION}

Conflict of interest: The authors declare that they have no competing interest.

\section{REFERENCES}

1. Mozaffarian D, Benjamin EJ, Go AS, Arnett DK, Blaha MJ, Cushman M, et al. Executive summary: heart disease and stroke statistics-2016 update: A Report From the American Heart Association. Circulation. 2016;133:447-54.

2. Gu WW, Lu SQ, Ni Y, Liu ZH, Zhou XY, Zhu YM, et al. 2-(3',5'-Dimethoxybenzylidene) cyclopentanone, a novel synthetic small-molecule compound, provides neuroprotective effects against ischemic stroke. Neuroscience. 2016;316:26-40.
3. O'Collins VE, Macleod MR, Donnan GA, Horky LL, van der Worp BH, Howells DW. 1026 experimental treatments in acute stroke. Ann Neurol. 2006;59:467-77.

4. Zaleska MM, Mercado ML, Chavez J, Feuerstein GZ, Pangalos MN, Wood A. The development of stroke therapeutics: promising mechanisms and translational challenges. Neuropharmacology. 2009;56:329-41.

5. Min JW, Lü L, Freeling JL, Martin DS, Wang H. USP14 inhibitor attenuates cerebral ischemia/reperfusion-induced neuronal injury in mice. J Neurochem. 2017;140: 826-33.

6. Zhu YM, Gao X, Ni Y, Li W, Kent TA, Qiao SG, et al. Sevoflurane postconditioning attenuates reactive astrogliosis and glial scar formation after ischemiareperfusion brain injury. Neuroscience. 2017;356:125-41.

7. Sofroniew MV, Vinters HV. Astrocytes: biology and pathology. Acta Neuropathol. 2010;119:7-35

8. Ding S. Dynamic reactive astrocytes after focal ischemia. Neural Regen Res. 2014;9:2048-52.

9. Faulkner JR, Herrmann JE, Woo MJ, Tansey KE, Doan NB, Sofroniew MV. Reactive astrocytes protect tissue and preserve function after spinal cord injury. J Neurosci. 2004;24:2143-55.

10. Silver J, Miller JH. Regeneration beyond the glial scar. Nat Rev Neurosci. 2004;5:146-56

11. Rolls A, Shechter R, Schwartz M. The bright side of the glial scar in CNS repair. Nat Rev Neurosci. 2009;10:235-41.

12. Wang $R$, Zhang $X$, Zhang J, Fan $Y$, Shen $Y$, Hu W, et al. Oxygen-glucose deprivation induced glial scar-like change in astrocytes. PLoS One. 2012;7:e37574.

13. Liu Z, Chopp M. Astrocytes, therapeutic targets for neuroprotection and neurorestoration in ischemic stroke. Prog Neurobiol. 2016;144:103-20.

14. Smith GM, Strunz C. Growth factor and cytokine regulation of chondroitin sulfate proteoglycans by astrocytes. Glia. 2005;52:209-18.

15. Gris $P$, Tighe A, Levin D, Sharma R, Brown A. Transcriptional regulation of scar gene expression in primary astrocytes. Glia. 2007;55:1145-55.

16. Karimi-Abdolrezaee S, Billakanti R. Reactive astrogliosis after spinal cord injurybeneficial and detrimental effects. Mol Neurobiol. 2012;46:251-64.

17. Sims NR, Yew WP. Reactive astrogliosis in stroke: contributions of astrocytes to recovery of neurological function. Neurochem Int. 2017;107:88-103.

18. Lipton P. Lysosomal membrane permeabilization as a key player in brain ischemic cell death: a "lysosomocentric" hypothesis for ischemic brain damage. Transl Stroke Res. 2013;4:672-84.

19. Rodríguez-Muela N, Hernández-Pinto AM, Serrano-Puebla A, García-Ledo L, Latorre $\mathrm{SH}$, de la Rosa EJ, et al. Lysosomal membrane permeabilization and autophagy blockade contribute to photoreceptor cell death in a mouse model of retinitis pigmentosa. Cell Death Differ. 2015;22:476-87.

20. Ni Y, Gu WW, Liu ZH, Zhu YM, Rong JG, Kent TA, et al. RIP1K contributes to neuronal and astrocytic cell death in ischemic stroke via activating autophagiclysosomal pathway. Neuroscience. 2018;371:60-74.

21. Johansson AC, Appelqvist $H$, Nilsson $C$, Kågedal $K$, Roberg $K$, Ollinger K. Regulation of apoptosis-associated lysosomal membrane permeabilization. Apoptosis. 2010;15:527-40.

22. Xu M, Yang L, Rong JG, Ni Y, Gu WW, Luo Y, et al. Inhibition of cysteine cathepsin $B$ and $L$ activation in astrocytes contributes to neuroprotection against cerebral ischemia via blocking the tBid-mitochondrial apoptotic signaling pathway. Glia. 2014;62:855-80.

23. Aits S, Jäättelä M. Lysosomal cell death at a glance. J Cell Sci. 2013;126:1905-12.

24. Hyttinen JM, Niittykoski M, Salminen A, Kaarniranta K. Maturation of autophagosomes and endosomes: a key role for Rab7. Biochim Biophys Acta. 2013;1833: 503-10.

25. Zhang M, Chen L, Wang S, Wang T. Rab7: roles in membrane trafficking and disease. Biosci Rep. 2009;29:193-209.

26. Yuan D, Liu C, Hu B. Dysfunction of membrane trafficking leads to ischemia-reperfusion injury after transient cerebral ischemia. Transl Stroke Res. 2018;9:1-8.

27. Hong LZ, Gu WW, Ni Y, Xu M, Yang L, Liu YL, et al. Postischemic long-term treatment with Qiangli Tianma Duzhong Capsule improves brain functional recovery via the improvement of hemorrheology and the inhibition of platelet aggregation in a rat model of focal cerebral ischemia. Evid-Based Complement Altern Med. 2013;2013:795365.

28. Qin AP, Liu CF, Qin YY, Hong LZ, Xu M, Yang L, et al. Autophagy was activated in injured astrocytes and mildly decreased cell survival following glucose and oxygen deprivation and focal cerebral ischemia. Autophagy. 2010;6:738-53.

29. He P, He W, Wang A, Xia T, Xu B, Zhang $M$, et al. PBDE-47-induced oxidative stress, DNA damage and apoptosis in primary cultured rat hippocampal neurons. Neurotoxicology. 2008;29:124-9.

30. Zinchuk V, Zinchuk O, Okada T. Quantitative colocalization analysis of multicolor confocal immunofluorescence microscopy images: pushing pixels to explore biological phenomena. Acta Histochem Cytochem. 2007;40:101-11. 
31. Sofroniew MV. Molecular dissection of reactive astrogliosis and glial scar formation. Trends Neurosci. 2009;32:638-47.

32. Burda JE, Sofroniew MV. Reactive gliosis and the multicellular response to CNS damage and disease. Neuron. 2014;81:229-48.

33. Choudhury GR, Ding S. Reactive astrocytes and therapeutic potential in focal ischemic stroke. Neurobiol Dis. 2016;85:234-44.

34. Wen H, Zhan L, Chen S, Long L, Xu E. Rab7 may be a novel therapeutic target for neurologic diseases as a key regulator in autophagy. J Neurosci Res. 2017;95:1993-2004.

35. Hayakawa K, Nakano T, Irie K, Higuchi S, Fujioka M, Orito K, et al. Inhibition of reactive astrocytes with fluorocitrate retards neurovascular remodeling and recovery after focal cerebral ischemia in mice. J Cereb Blood Flow Metab. 2010;30:871-82.

36. Barreto GE, Sun X, Xu L, Giffard RG. Astrocyte proliferation following stroke in the mouse depends on distance from the infarct. PLoS One. 2011;6:e27881.

37. Shimada IS, Borders A, Aronshtam A, Spees JL. Proliferating reactive astrocytes are regulated by Notch-1 in the peri-infarct area after stroke. Stroke. 2011:42:3231-7.

38. Bao Y, Qin L, Kim E, Bhosle S, Guo H, Febbraio M, et al. CD36 is involved in astrocyte activation and astroglial scar formation. J Cereb Blood Flow Metab. 2012;32:1567-77.

39. Lam T, Kulp DV, Wang R, Lou Z, Taylor J, Rivera CE, et al. Small molecule inhibition of Rab7 impairs B cell class switching and plasma cell survival to dampen the autoantibody response in murine lupus. J Immunol. 2016;197:3792-805.

40. Yuan D, Liu C, Hu B. Inactivation of NSF ATPase leads to cathepsin B release after transient cerebral ischemia. Transl Stroke Res. 2017;9:1-13.

41. Hill IE, Preston E, Monette R, MacManus JP. A comparison of cathepsin B processing and distribution during neuronal death in rats following global ischemia or decapitation necrosis. Brain Res. 1997;751:206-16.
42. Seyfried D, Han Y, Zheng Z, Day N, Moin K, Rempel S, et al. Cathepsin $B$ and middle cerebral artery occlusion in the rat. J Neurosurg. 1997;87: 716-23.

43. Benchoua A, Braudeau J, Reis A, Couriaud C, Onténiente B. Activation of proinflammatory caspases by cathepsin B in focal cerebral ischemia. J Cereb Blood Flow Metab. 2004;24:1272-9.

44. Kilinc M, Gürsoy-Ozdemir Y, Gürer G, Erdener SE, Erdemli E, Can A, et al. Lysosomal rupture, necroapoptotic interactions and potential crosstalk between cysteine proteases in neurons shortly after focal ischemia. Neurobiol Dis. 2010;40:293-302.

45. Zhou XY, Luo Y, Zhu YM, Liu ZH, Kent TA, Rong JG, et al. Inhibition of autophagy blocks cathepsins-tBid-mitochondrial apoptotic signaling pathway via stabilization of lysosomal membrane in ischemic astrocytes. Cell Death Dis. 2017; 8:e2618.

46. Hsu KF, Wu CL, Huang SC, Wu CM, Hsiao JR, Yo YT, et al. Cathepsin L mediates resveratrol-induced autophagy and apoptotic cell death in cervical cancer cells. Autophagy. 2009;5:451-60.

47. Pekny M, Pekna M. Astrocyte reactivity and reactive astrogliosis: costs and benefits. Physiol Rev. 2014;94:1077-98.

48. Lukovic D, Stojkovic M, Moreno-Manzano V, Jendelova P, Sykova E, Bhattacharya SS, et al. Concise review: reactive astrocytes and stem cells in spinal cord injury: good guys or bad guys. Stem Cells. 2015;33:1036-41.

49. Buffo A, Rolando C, Ceruti S. Astrocytes in the damaged brain: molecular and cellular insights into their reactive response and healing potential. Biochem Pharmacol. 2010;79:77-89.

50. Zhan L, Chen S, Li K, Liang D, Zhu X, Liu L, et al. Autophagosome maturation mediated by Rab7 contributes to neuroprotection of hypoxic preconditioning against global cerebral ischemia in rats. Cell Death Dis. 2017;8:e2949. 